\title{
Layered THz waveguides for SPPs, filter and sensor applications
}

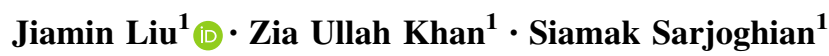

Received: 25 February 2019/Accepted: 1 November 2019/Published online: 12 November 2019

(C) The Author(s) 2019

\begin{abstract}
Theory of five kinds of layered structure $\mathrm{THz}$ waveguides is presented. In these waveguides, the modified and hybrid $\mathrm{THz}$ surface plasmon-polaritons (SPPs) are researched in detail. On these modes, the effects of material in each layer are discussed. The anti-resonant reflecting mechanism is also discussed in these waveguides. The mode characteristics of both TM mode and TE mode are analyzed for guiding TM mode with low loss and TE modes with huge loss in one waveguide: the TE modes filter application is put forward. The mode characteristics for one waveguide have useful sensor applications: for $\mathrm{TE}_{1}$ mode, we find that the low cut-off frequency has a sensitivity $(S)$ to the refractive index of the dielectric slab. The highest $S$ can be $666.7 \mathrm{GHz} / \mathrm{RIU}$ when $n_{2}=1.5, w=0$ and $t=0.1 \mathrm{~mm}$. We believe these results are very useful for designing practical $\mathrm{THz}$ devices for SPPs, filter and sensor applications.
\end{abstract}

Keywords THz waveguides $\cdot$ Surface plasmon-polaritons · Filters · Sensors

Jiamin Liu

jiamin.liu@qmul.ac.uk

$\bowtie$ Siamak Sarjoghian

s.sarjoghian@qmul.ac.uk

Zia Ullah Khan

zia.khan@qmul.ac.uk

1 School of Electronic Engineering and Computer Science, Queen Mary University of London, London E1 4NS, UK

\section{Introduction}

Terahertz $(\mathrm{THz})$ wave as surface plasmon-polaritons (SPPs) has attracted extensive attention over the last several years [1-3]. THz SPPs can be guided on metal wires $[1,4,5]$, metal plates [2, 6-8] or metal films [3]. Wang and Mittleman reported the first THz SPPs on metal wires with low losses (experimental $1 \mathrm{~m}^{-1}$ ) and negligible group velocity dispersion (GVD) [1, 4]. Before Wang's work, the SPP mode is only researched on optical ranges. Researchers have followed to study THz SPPs on metal wires. The basic demonstration is based on the theory presented by Sommerfeld in 1899 [9]. In recent years, the modified SPP modes in optical ranges are discussed in several papers [10-12]. However, the modified SPPs in $\mathrm{THz}$ range are still not discussed particularly.

The hybrid plasmonic modes in optics are also researched in several papers $[13,14]$. This mode is a hybrid mode of the SPP mode and dielectric mode, which makes the hybrid mode with lower loss than the SPP mode and better confinement than the dielectric mode. However, the hybrid SPP mode in $\mathrm{THz}$ range is only discussed by a few papers [2] particularly. The hybrid $\mathrm{THz}$ SPP mode on doubledielectric-slab-coated metal plate waveguide (DDSCMPW) has not been discussed yet.

For ordinary $\mathrm{THz}$ waveguides, planar waveguides [15-17] are acting important roles in many applications because of their simple structure [17], low loss [18], high coupling efficiency [19] and good confinement [20]. Planar $\mathrm{THz}$ waveguides, such as parallel-plate waveguides [18, 21-23], metal film-coated double-dielectric-slab waveguides [20], single metal plate waveguides [2], and metal-clad hollow waveguides [24, 25] are reported. Antiresonant reflecting mechanism is discovered in the plasticcoated parallel-plate waveguide [18] and metal-clad hollow 
waveguides [24, 25]. And applications of tunable terahertz filters based on planar structures are reported by many papers [20, 23-26].

In this paper, we present five kinds of layered $\mathrm{THz}$ waveguides. In Sect. 2, all the structures and the dispersion equations are given. In Sect. 3, the modified THz SPPs in the dielectric-slab-coated metal film waveguide (DSCMFW) is discussed. In Sect. 4, the triple-layer-dielectric-slab waveguide (TLDSW) with high-low-high refractive indices is discussed. In Sect. 5, the mode characteristics of the metal nanofilm-dielectric-plate waveguide (MNDPW) of both TM mode and TE mode are discussed. We put forward the application of a TE modes filter of this waveguide. In Sect. 6, we discuss the mode characteristics in the single-dielectric-slab-coated parallel-plate waveguide (SDSCPPW). In Sect. 7, the hybrid THz SPPs on the double-dielectric-slab-coated metal plate waveguide (DDSCMPW) is presented. At the resonant frequencies, the hybrid SPP modes transfer to dielectric modes. However, for the high-low indices coating, the hybrid mode is always a SPP-like mode. To the best of our knowledge, these layered $\mathrm{THz}$ waveguides are still not researched in detail and we believe these results are useful in designing $\mathrm{THz}$ waveguides and the according applications such as $\mathrm{THz}$ SPPs, filters and sensors. slab-coated parallel-plate waveguide (SDSCPPW), and (e) is the double-dielectric-slab-coated metal plate waveguide (DDSCMPW). They are four-layer structures.

The material of each layer is shown in the figure. $n_{1}, n_{2}$ and $n_{3}$ are the refractive index accordingly. In Fig. 1a, $d$ is the thickness of the coating dielectric slab with a refractive index of $n_{2}$, and $t$ is the thickness of the metal film with a refractive index of $n_{1}$. In Fig. 1b, the materials of the two coating slabs are the same and with a refractive index of $n_{2}$ and a thickness of $t$. The middle dielectric slab has a different material with a refractive index of $n_{1}$ and a width of $w$. In Fig. 1c, the middle metal film has a thickness of $t$ and a refractive index of $n_{1}$; the two-coated dielectric slabs have a thickness of $d$ and a refractive index of $n_{2}$; and the outside metal plates have a refractive index of $n_{1}$. In Fig. $1 \mathrm{~d}, t$ is the thickness of the dielectric slab, $w$ is the width of the air gap.

The TM mode and TE mode of the THz wave are guided in $z$-direction; the width in $y$-direction is large enough. This is the well-known two-dimensional analysis [27]. The field equations of the even guiding mode in each layer are similar for the first three five-layer structures. Take the metal nanofilm-dielectric-plate waveguides (MNDPW) as an example, the mode field equations can be written as $[18,19,28]$ :

The dispersion equations of the guiding modes (five-layered) can be written as $[18,19,28]$ :

TM mode: $\quad H_{y}(x)= \begin{cases}A \cos \left(h_{1} x\right) & |x| \leq \frac{t}{2} \\ A\left[\cos \left(h_{1} \frac{t}{2}\right) \cos h_{2}\left(|x|-\frac{t}{2}\right)-\frac{h_{1} \varepsilon_{2}}{h_{2} \varepsilon_{1}} \sin \left(h_{1} \frac{t}{2}\right) \sin h_{2}\left(|x|-\frac{t}{2}\right)\right] & \frac{t}{2} \leq|x| \leq \frac{t}{2}+d \\ A\left[\cos \left(h_{1} \frac{t}{2}\right) \cos h_{2} d-\frac{h_{1} \varepsilon_{2}}{h_{2} \varepsilon_{1}} \sin \left(h_{1} \frac{t}{2}\right) \sin h_{2} d\right] e^{-h_{3}\left(|x|-\frac{t}{2}-d\right)} & |x| \geq \frac{t}{2}+d\end{cases}$

TE mode: $\quad E_{y}(x)= \begin{cases}A \cos \left(h_{1} x\right) & |x| \leq \frac{t}{2} \\ A\left[\cos \left(h_{1} \frac{t}{2}\right) \cos h_{2}\left(|x|-\frac{t}{2}\right)-\frac{h_{1}}{h_{2}} \sin \left(h_{1} \frac{t}{2}\right) \sin h_{2}\left(|x|-\frac{t}{2}\right)\right] & \frac{t}{2} \leq|x| \leq \frac{t}{2}+d \\ \left.A\left[\cos \left(h_{1} \frac{t}{2}\right) \cos h_{2} d-\frac{h_{1}}{h_{2}} \sin \left(h_{1} \frac{t}{2}\right) \sin h_{2} d\right]\right] e^{-h_{3}\left(|x|-\frac{t}{2}-d\right)} & |x| \geq \frac{t}{2}+d\end{cases}$

\section{The waveguide structures and the dispersion equations}

The structures of these layered $\mathrm{THz}$ waveguides are shown in Fig. 1: (a) is the dielectric-slab-coated metal film waveguide (DSCMFW); (b) is the triple-layer-dielectricslab waveguide (TLDSW); (c) is the metal nanofilm-dielectric-plate waveguides (MNDPW). They are all fivelayer symmetrical structures. (d) is the single-dielectric-
TM mode: $\quad h_{1} \frac{t}{2}=\tan ^{-1}\left[\frac{1-\frac{h_{2} \varepsilon_{3}}{h_{3} \varepsilon_{2}} \tan h_{2} d}{\frac{h_{1} \varepsilon_{3}}{h_{3} \varepsilon_{1}}+\frac{h_{1} \varepsilon_{2}}{h_{2} \varepsilon_{1}} \tan h_{2} d}\right]$

TE mode: $\quad h_{1} \frac{t}{2}=\tan ^{-1}\left[\frac{1-\frac{h_{2}}{h_{3}} \tan h_{2} d}{\frac{h_{1}}{h_{3}}+\frac{h_{1}}{h_{2}} \tan h_{2} d}\right]$

where $\quad h_{1}=\left(n_{1}^{2} \quad k^{2}-\beta^{2}\right)^{1 / 2}, h_{2}=\left(n_{2}^{2} k^{2}-\right.$ $\left.\beta^{2}\right)^{1 / 2}, h_{3}=\left(\beta^{2}-n_{1}^{2} k^{2}\right)^{1 / 2} ; \beta=\beta_{1}-j * \alpha$ is the complex 


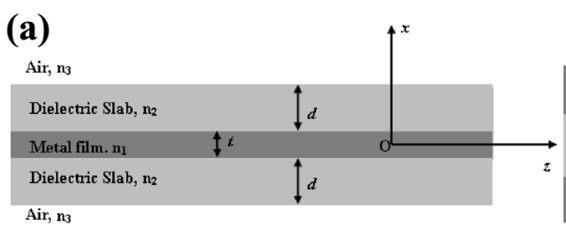

(d) (b)

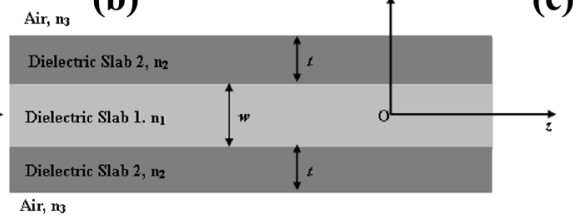

(c)

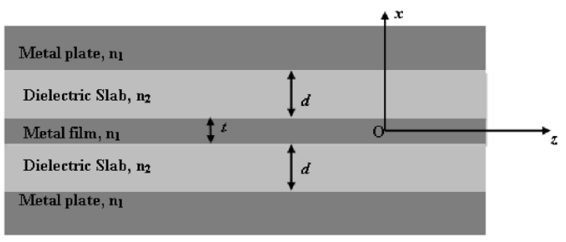

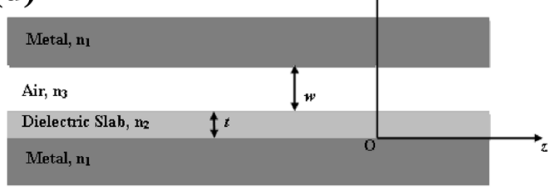

Fig. 1 The structures of these five kinds of layered $\mathrm{THz}$ waveguides

variable, in which the real part $\beta_{1}$ is related to the effective refractive index $\left(n_{\mathrm{eff}}=\beta_{1} / k\right)$ and the imaginary part $\alpha$ is the amplitude loss coefficient of the guiding mode. $k=$ $2 \pi / \lambda$ is the wave vector in vacuum. $\varepsilon_{1}=n_{1}^{2}$ and $\varepsilon_{2}=n_{2}^{2}$ are relative permittivities of the metal and dielectric slab, respectively.

For structures in Fig. 1a, b, Eqs. (1)-(4) only need slightly changes, for example, we should use $h_{3}=\left(\beta^{2}-\right.$ $\left.\varepsilon_{3} k_{0}^{2}\right)^{1 / 2}$ and the according refractive index of the according materials for (a) and use $w$ to replace $t, t$ replace $d$ for (b).

In similar way, the dispersion equations of the guiding modes in the single-dielectric-slab-coated parallel-plate waveguide (SDSCPPW) (4-layered, Fig. 1d) can be written as $[2,28]$ :

TM mode: $\quad \tan h_{2} t=\frac{\frac{2 h_{1}}{\varepsilon_{1}}+\left(\frac{h_{1}^{2} \varepsilon_{3}}{h_{3} \varepsilon_{1}^{2}}-\frac{h_{3}}{\varepsilon_{3}}\right) \tan h_{3} w}{\left(\frac{h_{3} h_{1} \varepsilon_{2}}{h_{2} \varepsilon_{1} \varepsilon_{3}}+\frac{h_{1} h_{2} \varepsilon_{3}}{h_{3} \varepsilon_{2} \varepsilon_{1}}\right) \tan h_{3} w+\frac{h_{2}}{\varepsilon_{2}}-\frac{h_{1}^{2} \varepsilon_{2}}{h_{2} \varepsilon_{1}^{2}}}$

(e)

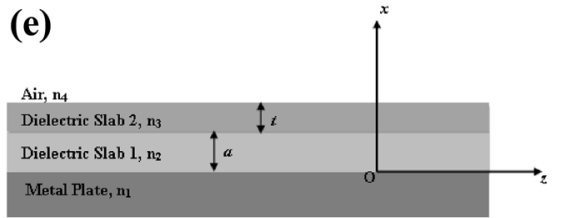

As shown in Fig. 1e, we can see the structure of the double-dielectric-slab-coated metal-plate waveguide (DDSCMPW) has four layers: the first layer is the metal plate with a refractive index of $n_{1}$; the second layer is dielectric slab 1 with a refractive index of $n_{2}$ and a thickness of $a$; the third layer is dielectric slab 2 with a refractive index of $n_{3}$ and a thickness of $t$; the fourth layer is the outside air with a refractive index of $n_{4}$. The waveguide has a low-high refractive indices (1-h) coating $\left(n_{2}<n_{3}\right)$ structure and a high-low refractive indices (h-l) coating $\left(n_{2}>n_{3}\right)$ structure. The hybrid THz SPPs modes are transverse magnetic (TM) polarized $\mathrm{THz}$ waves and transmit in $z$-direction, the width in $y$-direction is large enough. The mode field equations of the guiding mode in each layer can be written as $[2,28]$ :

As the tangential components of the electromagnetic fields on interfaces are continuous, we can obtain the dispersion equation of the guiding TM mode $[2,28]$ :

TE mode: $\quad \tan h_{2} t=\frac{2 h_{1}+\left(\frac{h_{1}^{2}}{h_{3}}-h_{3}\right) \tan h_{3} w}{\left(\frac{h_{3} h_{1}}{h_{2}}+\frac{h_{1} h_{2}}{h_{3}}\right) \tan h_{3} w+h_{2}-\frac{h_{1}^{2}}{h_{2}}}$

$x \geq a+t$

$a \leq x \leq a+t$

$0 \leq x \leq a$

$x \leq 0$ 
$\tan h_{2} a=\frac{\frac{h_{4}}{\varepsilon_{4}}+\frac{h_{1}}{\varepsilon_{1}}+\left(\frac{h_{4} h_{1} \varepsilon_{3}}{h_{3} \varepsilon_{1} \varepsilon_{4}}-\frac{h_{3}}{\varepsilon_{3}}\right) \tan h_{3} t}{\left(\frac{h_{3} h_{1} \varepsilon_{2}}{h_{2} \varepsilon_{1} \varepsilon_{3}}+\frac{h_{4} h_{2} \varepsilon_{3}}{h_{3} \varepsilon_{2} \varepsilon_{4}}\right) \tan h_{3} t+\frac{h_{2}}{\varepsilon_{2}}-\frac{h_{4} h_{1} \varepsilon_{2}}{h_{2} \varepsilon_{1} \varepsilon_{4}}}$

By dispersion Eqs. (3), (4), (5), (6) and (8), we can calculate the propagation constant $\beta$ of the waveguide for TM mode and TE mode, respectively. Pursuantly, we can get the loss coefficients and effective refractive indices of these patterns. Further, according to the propagation constant and Eqs. (1), (2), and (7), we can find out the field distributions of mode patterns.

\section{Modified THz SPPs on dielectric-slab-coated metal film waveguide (DSCMFW)}

The material of the metal is chosen as copper for all the cases. For this case, the waveguide structure is shown in Fig. 1a. The parameter of copper film in $\mathrm{THz}$ region can be gotten from the Drude mode [29]:

$\varepsilon_{3}=\varepsilon_{\infty}-\frac{\omega_{p}^{2}}{\omega^{2}-i \omega \omega_{\tau}}$

where $\varepsilon_{\infty}$ is the high frequency permittivity of copper which is always negligible in $\mathrm{THz}$ region, $\omega_{\mathrm{p}-}$ $=1.1234 * 10^{16} \mathrm{rad} / \mathrm{s}$ [29] is the plasma oscillation frequency of copper, $\omega_{\tau}=1.3798 * 10^{13} \mathrm{rad} / \mathrm{s}$ [29] is the damping frequency of copper, and $\omega$ is the angular frequency of the $\mathrm{THz}$ wave.

The material of the dielectric slab is chosen as the silicon here, in Drude mode: $\varepsilon_{\infty}=11.7$ is the high frequency dielectric constant. We adopt the doped silicon which has parameters of $\omega_{\mathrm{p}}=0.01 * 10^{12} \mathrm{rad} / \mathrm{s}, \quad \omega_{\tau}=0.67 * 10^{12}$ $\mathrm{rad} / \mathrm{s} . \varepsilon_{1}=n_{1}^{2}=1$ is the relative permittivity of air. The modified plasmonic modes are transverse magnetic (TM) polarized $\mathrm{THz}$ waves.

We calculate Eq. (3) at the condition of $t=10 \mathrm{~nm}$ and $d=0.1 \mathrm{~mm}$, and get the changing law of guiding mode loss (solid line) to $\mathrm{THz}$ frequency $f$, as shown in Fig. 2a. The corresponding effective refractive index (dashed line) and group velocity $\left(\frac{v_{\mathrm{g}}}{c}=\frac{1}{n_{\text {eff }}\left(1+\frac{k_{0} \mathrm{~d}_{n_{\mathrm{eff}}}}{n_{\text {eff }} \mathrm{d}_{k_{0}}}\right)}[18]\right.$, dotted line) are also shown.

From Fig. 2a, we can see that the law of loss to $f$ has three segments. In the region of $0.1-0.23 \mathrm{THz}$, the loss increases sharply as $f$ increases. The changing slopes of effective refractive index and group velocity to $f$ are much sharper in this region which tells us that both the phase velocity dispersion (PVD) and the group velocity dispersion (GVD) are much higher in this region. When the $\mathrm{THz}$ frequency is very low, the wavelength is much larger, and the diffraction limit of the $\mathrm{THz}$ wave makes the beam width larger. As the waveguide size is fixed here, the metal film and the coated silicon slab cannot cause too much loss. In this range as the frequency increases, the mode confinement will be better and better, and the effects of the metal film and the coated silicon slab on the mode will become more and more important.

In the region of $0.23-0.48 \mathrm{THz}$, the loss decreases as $f$ increases, and both the PVD and GVD are lower, which tells us that the effects of metal film are lower. This is because more energy will be confined in the coated slabs. According to our calculation by Drude mode, the loss caused by silicon decreases as $f$ increase in this region.

In the region of higher than $0.48 \mathrm{THz}$, the loss increases monotonously and the dispersions are almost zero. When the $\mathrm{THz}$ wavelength becomes even lower, the confinement of the mode will make even more energy interact with the metal film. The loss of this dielectric-coated film is extreme low (lower than $0.016 \mathrm{~m}^{-1}$ ), which is one order of magnitude lower than the theoretical loss of THz SPPs on metal wires [5]. This is because the metal film is much thinner than the penetration depth of $\mathrm{THz}$ waves to metal wires (about $100 \mathrm{~nm}$ ) even though the $\mathrm{THz}$ wavelength is the same as Ref. [5].

According to Eq. (1), we get the mode field at $0.25 \mathrm{THz}$ (solid line) and $0.5 \mathrm{THz}$ (dashed line) at the condition of $t=10 \mathrm{~nm}$ and $d=0.1 \mathrm{~mm}$, as shown in Fig. 2b. We can see that the mode energy is almost concentrated in the
Fig. 2 a The guiding mode loss (solid line), effective refractive index (dashed line) and group velocity (dotted line) of the dielectric-coated metal film waveguide as a function of $\mathrm{THz}$ frequency $f$ at the condition of $t=10 \mathrm{~nm}$ and $d=0.1 \mathrm{~mm}$, b the corresponding mode field distribution at $f=0.25 \mathrm{THz}$ (solid line) and $f=0.5 \mathrm{THz}$ (dashed line)

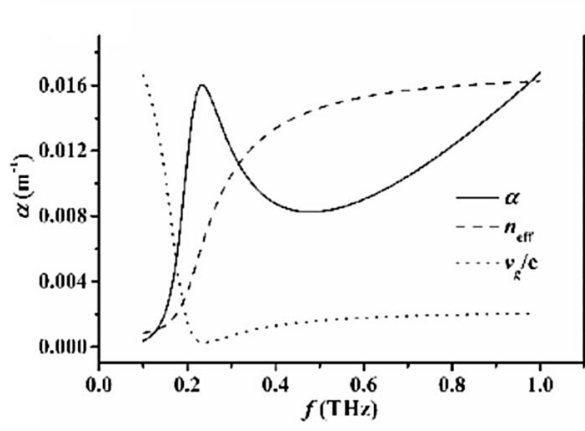

(a)
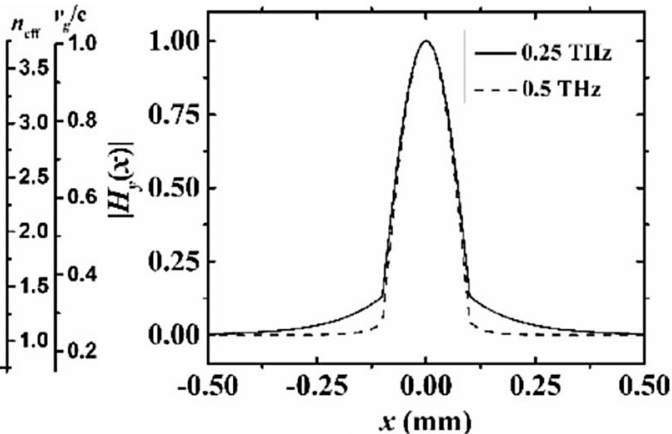

(b) 
Fig. 3 a The changing law of mode loss (solid line) and effective refractive index (dashed line) of the modified SPP mode to the coated slab thickness $d$ at the condition of $t=10 \mathrm{~nm}$ and $f=0.5 \mathrm{THz}$. b The changing law of mode loss to the thickness $t$ of the metal film at the condition of $d=0.1 \mathrm{~mm}$ and $f=0.5 \mathrm{THz}$

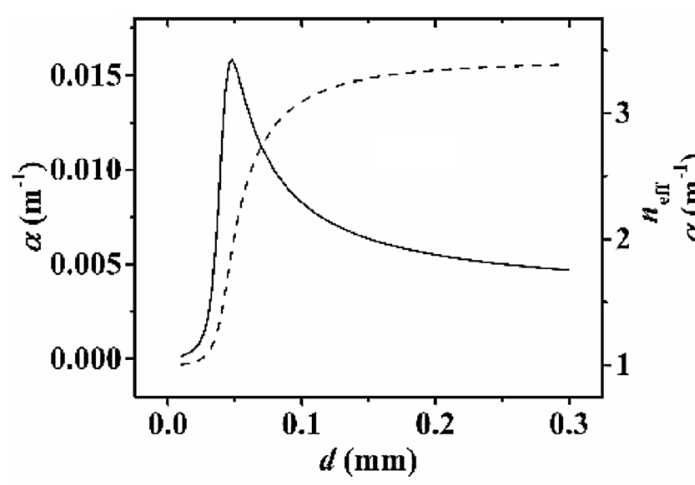

(a)

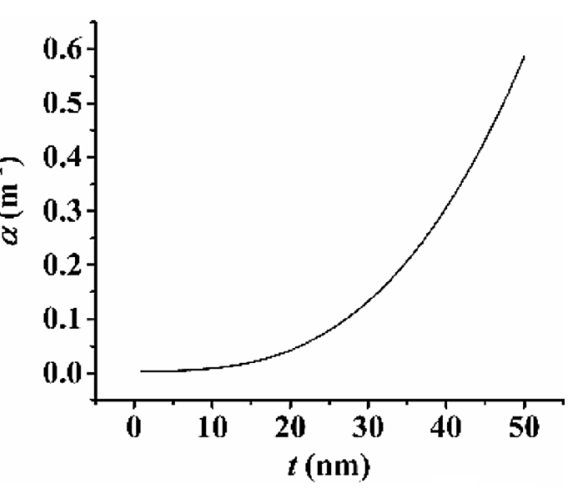

(b) dielectric slab and the beam width is only about $0.2 \mathrm{~mm}$, while the wavelength of $\mathrm{THz}$ wave is $1.2 \mathrm{~mm}$ at $0.25 \mathrm{THz}$. The degree of the energy concentration is higher at the larger $f$, as can be seen the mode field at $0.5 \mathrm{THz}$ (dashed line) in Fig. 2b. For $\mathrm{THz}$ metal wires, the mode field amplitude is still strong at the distance of $10 \mathrm{~mm}$ [5] from the metal wire surface at the same condition.

The silicon coating makes the confinement of this modified SPP mode much better. After our calculation, we find that the pure SPP mode on this metal film is very leaky, the mode confinement is too weak and the mode cannot be guided effectively, because the wavelength of $\mathrm{THz}$ wave is much larger than the thickness of the metal film, the diffraction limit makes almost no electric-matter interaction between TM mode and the much thinner metal film.

The loss and effective refractive index of modified $\mathrm{THz}$ SPP mode on this waveguide are gotten as a function of $d$, as shown in Fig. 3a.

The loss is extreme low when the thickness $d$ of the slab is much thinner. However, in that case, the effective refractive index is almost 1 , most of the energy is in the air and the beam width is much larger. When $d$ increases, the loss has a maximum point and then decreases significantly.
The effective refractive index increases monotonously to that of the silicon, the energy will be distributed more in the dielectric slab, and the beam width will be smaller. The loss is lower for larger $d$. So the larger the $d$ is, the longer the THz waves can transmit and the smaller the beam width is. This is because the effect of the slab on the mode is more obvious for larger $d$.

We also get the loss changing as a function of metal film thickness $t$, as shown in Fig. 3b. The loss of the modified $\mathrm{THz}$ SPP mode increases monotonously as $t$ increases. The effects of metal film to the modified THz SPPs will be obviously larger when $t$ is larger.

\section{THz triple-layer-dielectric-slab waveguide (TLDSW) with anti-resonant reflecting}

The waveguide structure is shown in Fig. 1b. The waveguide has high-low-high refractive indices $\left(n_{1}<n_{2}\right)$ here. Silicon with $\operatorname{Real}\left[n_{2}\right]=\sqrt{11.7}=3.42$ and an absorption loss by Drude model (as seen above) is chosen as $n_{2}$. Polystyrene (PS) with a parameter of $n_{1}=\sqrt{\varepsilon_{1}}=1.58-$ $j 0.0036$ [30] is chosen as $n_{1}$. The absorption loss of the PS

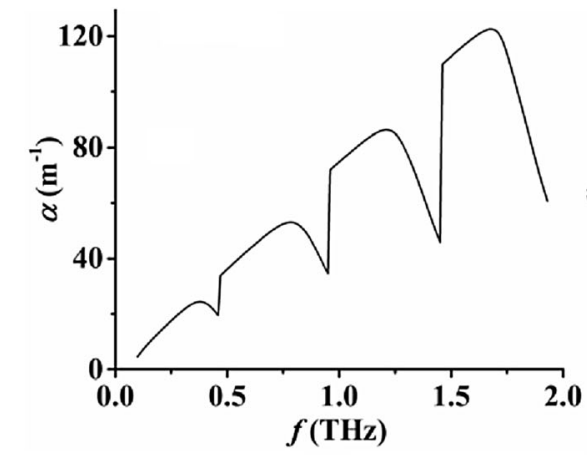

(a)

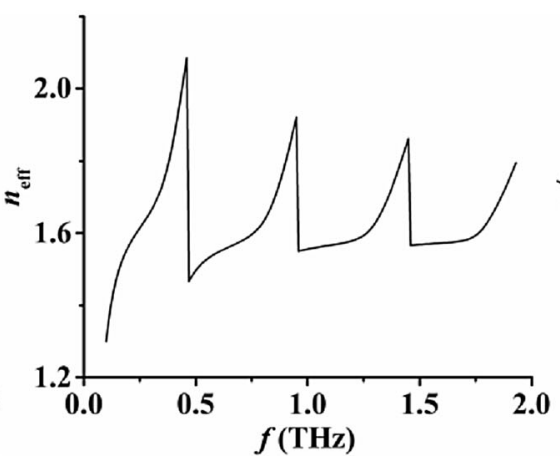

(b)

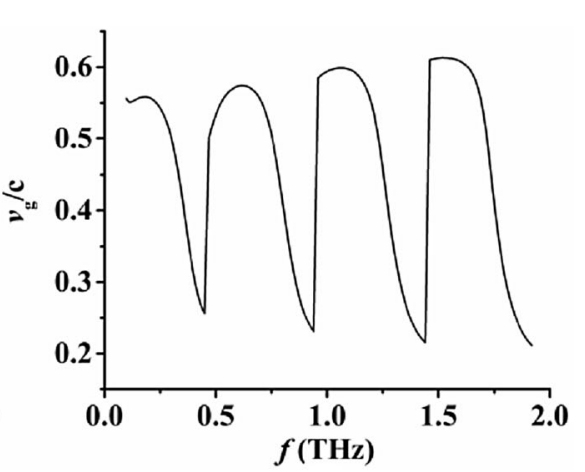

(c)

Fig. 4 The loss (a), effective refractive index (b) and group velocity (c) of the triple-layer-dielectric-slab waveguide with high-low-high refractive indices change as a function of THz frequency $f$ when $t=0.1 \mathrm{~mm}, w=0.5 \mathrm{~mm}$ 
is much larger than that of the silicon. We only consider TM mode here.

By Eq. (3), the loss, effective refractive index and group velocity are gotten as a function of the $\mathrm{THz}$ wave frequency $f$, as shown in Fig. 4.

The loss of the guiding mode appears nadirs at the frequencies of $0.46 \mathrm{THz}, 0.95 \mathrm{THz}$ and $1.45 \mathrm{THz}$. They are caused by the anti-resonant reflection mechanism. The loss nadir points can be predicted by the following relation, namely the relation of the resonant frequency [18]:

$f_{m}=\frac{m \mathrm{c}}{2 t \sqrt{n_{2}^{2}-n_{1}^{2}}}$

$m=1,2,3 \ldots$ is the resonance order. We substitute $c=3 * 10^{8} \mathrm{~m} / \mathrm{s}, t=0.1 \mathrm{~mm}, n_{2}=3.42$ and $n_{1}=1.58$ to Eq. (10) and get $f_{1}=0.49 \mathrm{THz}, f_{2}=0.99 \mathrm{THz}$ and $f_{3}=1.48 \mathrm{THz}$. The numerical calculation values appear a little redshift comparing with the theory values, which is caused by the approximating of Eq. (10).

When $f$ is at the resonant frequencies, the energy of $\mathrm{THz}$ wave will transmit through the interfaces between the core PS and the coated silicon, and more energy will distribute in the lower loss silicon. However, anti-resonant reflections happen when $f$ is not the resonant frequencies and most of the energy will be confined in the high loss PS slab. In Fig. 4b, c, the effective refractive index of the guiding mode appears peaks and the group velocity of the guiding mode appears nadirs.

In order to get a better understanding of the mode in the waveguide with the anti-resonant guiding mechanism, we calculate the mode field of the guiding mode at the antiresonant frequency $0.96 \mathrm{THz}$ and the resonant frequency $1.45 \mathrm{THz}$ when $t=0.1 \mathrm{~mm}$ and $w=0.5 \mathrm{~mm}$, as shown in Fig. 5.

Figure 5 shows the evidence of less energy distributing in lower loss silicon at the anti-resonant frequency $(0.96 \mathrm{THz})$ and more at the resonant frequency $(1.45 \mathrm{THz})$. We can also see that the phase in one coating silicon slab is about $2 \pi$ at $0.96 \mathrm{THz}$ (the start frequency of resonant mode order $m=3$ ) and $3 \pi$ at $1.45 \mathrm{THz}$ (resonant order $m=3$ ), which has been discussed in Ref. [18].

At $f=1.0 \mathrm{THz}$ and $w=0.5 \mathrm{~mm}$, the loss (a) and the corresponding effective refractive index (b) of the guiding mode are gotten as a function of $t$, as shown in Fig. 6 .

When the thicknesses of the silicon slabs are close to $0.044 \mathrm{~mm}, 0.096 \mathrm{~mm}, 0.146 \mathrm{~mm}$ and $0.194 \mathrm{~mm}$, the loss coefficient decreases sharply. When $1.0 \mathrm{THz}$ becomes the resonant frequency for the coated silicon thickness, much more energy will distribute in the low loss silicon, and the effective refractive index will also be larger as can be seen in Fig. 6b. The resonant silicon thicknesses can be predicted by the following relation [18]:

$t_{m}=\frac{m c}{2 f \sqrt{n_{2}^{2}-n_{1}^{2}}}$

We substitute $c=3 * 10^{8} \mathrm{~m} / \mathrm{s}, f=1.0 \mathrm{THz}, n_{2}=3.42$ and $n_{1}=1.58$ to Eq. (11), and get $t_{1}=0.049 \mathrm{~mm}$, $t_{2}=0.099 \mathrm{~mm}, t_{3}=0.148 \mathrm{~mm}$ and $t_{4}=0.198 \mathrm{~mm}$. The numerical values are in good agreement as the theoretical values.

When $f=1.0 \mathrm{THz}$ and $t=0.1 \mathrm{~mm}$, the loss and the corresponding effective refractive index of the guiding mode are gotten as a function of $w$, as shown in Fig. 7.

Both the loss and the effective refractive index (to that of PS) are increasing monotonously. When $w$ is larger, the energy of the $\mathrm{THz}$ wave will distribute more in the high loss PS slab and less in the low loss silicon and outside air. According to our calculation, if chosen $n_{1}>n_{2}$ the mode will show total internal reflecting (TIR) which is not so interesting.

\section{THz wave in metal nanofilm-dielectric-plate waveguide (MNDPW)}

The waveguide structure is shown in Fig. 1c. The material of the metal nanofilm and the metal plate is chosen as copper. The material of the two-coated dielectric slabs is
Fig. 5 The mode field distribution at the anti-resonant frequency $0.96 \mathrm{THz}(\mathbf{a})$ and the resonant frequency $1.45 \mathrm{THz}$ (b) when $t=0.1 \mathrm{~mm}$ and $w=0.5 \mathrm{~mm}$

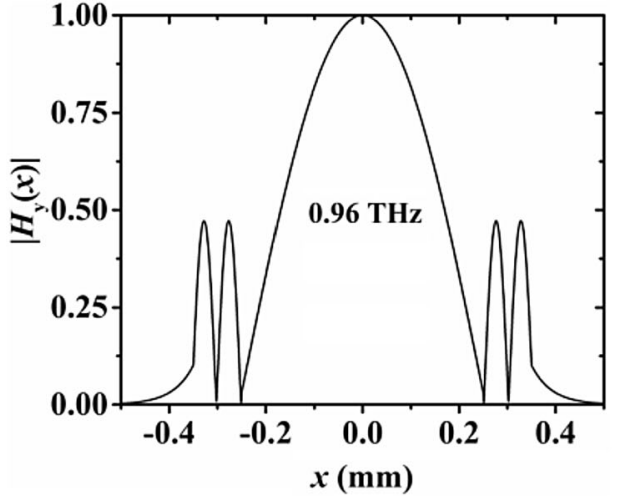

(a)

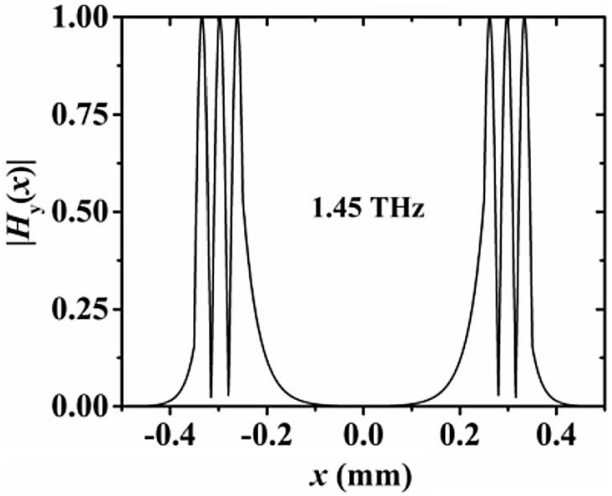

(b) 
Fig. 6 The laws of loss (a) and the corresponding effective refractive index (b) of the guiding mode changing as a function of $t$ when $f=1.0 \mathrm{THz}$ and $w=0.5 \mathrm{~mm}$

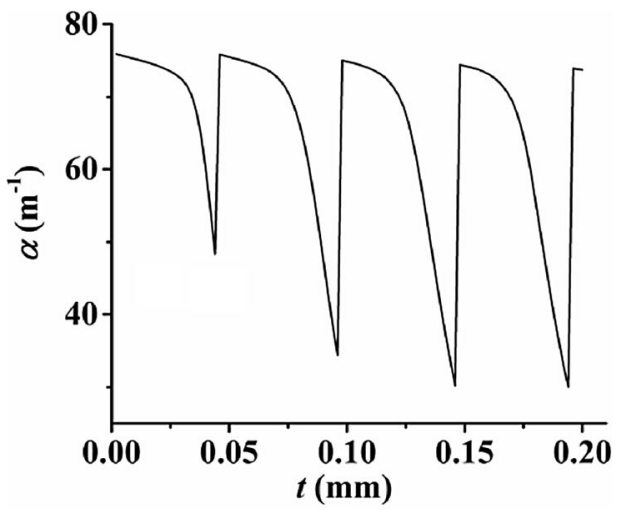

(a)

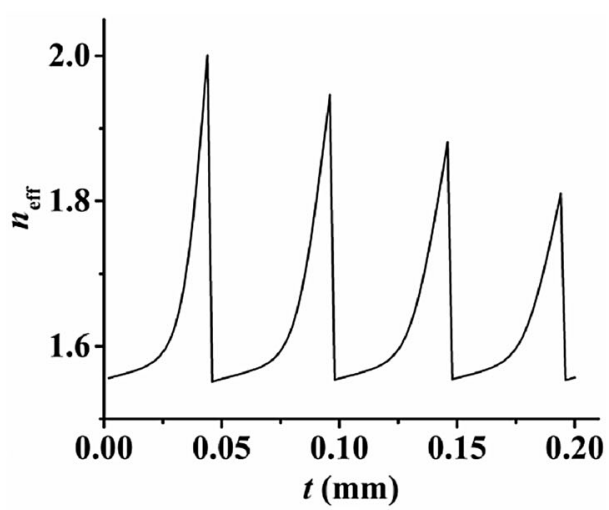

(b)

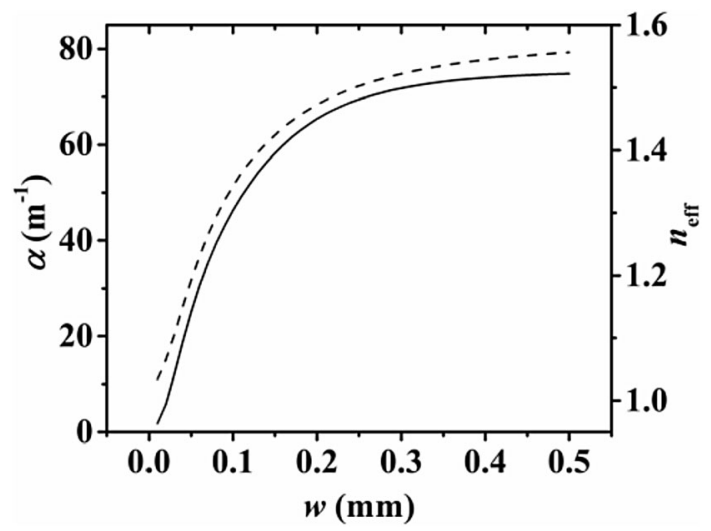

Fig. 7 The law of loss (solid line) of the guiding mode changing as a function of $w$ when $f=1 \mathrm{THz}, t=0.1 \mathrm{~mm}$. The dashed line is the corresponding effective refractive index of the guiding mode

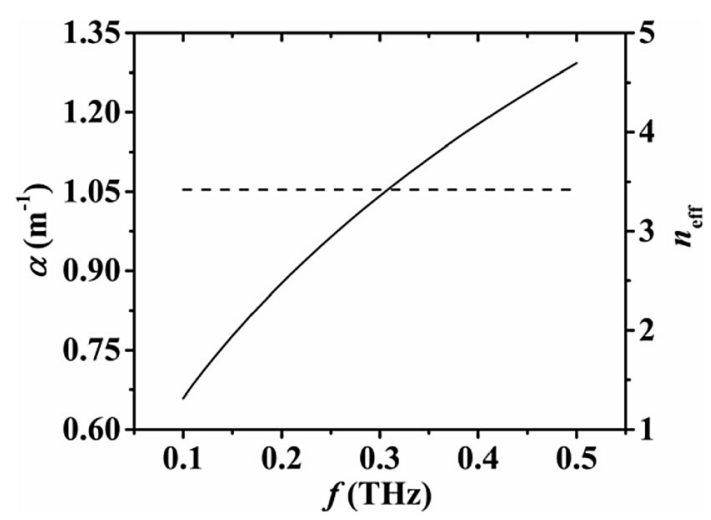

Fig. 8 The loss (solid line) and the corresponding effective refractive index (dashed line) of TM mode in the waveguide change as a function of THz frequency $f$ when $t=10 \mathrm{~nm}$ and $d=0.5 \mathrm{~mm}$

chosen as silicon. The parameters of copper and silicon in $\mathrm{THz}$ region are seen above. We consider both TM and TE mode here.

When the thickness of the nanofilm is $t=10 \mathrm{~nm}$ and the thickness of the two-coated dielectric slabs is $d=0.5 \mathrm{~mm}$, we calculate Eq. (3) and get the loss (solid line) and the corresponding effective refractive index (dashed line) of $\mathrm{TM}$ mode in the waveguide as a function of $\mathrm{THz}$ frequency $f$, as shown in Fig. 8.

The loss of the TM mode of the waveguide increases monotonously as $f$ increases. In the range of $0.1-0.5 \mathrm{THz}$, the loss is below $1.29 \mathrm{~m}^{-1}$ and at $0.1 \mathrm{THz}$ the loss can be as low as $0.66 \mathrm{~m}^{-1}$. The effective refractive index of the mode is always about 3.42, the mode is based on most of the energy propagates in the coated silicon slabs. The slope of the effective refractive index to the frequency is almost zero which tells us the GVD of the TM mode is almost 0 . This is because in the waveguide, only the electric field amplitude will have interaction with the metal. However, the electric field amplitude in TM mode is very small. So the interaction of TM mode with metal in this waveguide is very small.

We calculate Eq. (4) and get the losses (a) and the corresponding effective refractive indices (b) of TE modes in the waveguide as a function of THz frequency $f$ when $t=10 \mathrm{~nm}$ and $d=0.5 \mathrm{~mm}$, as shown in Fig. 9 .

Multiple TE modes appear in the waveguide since the losses and the effective refractive indices are discontinuous at every frequency. The losses of the TE modes can be as high as $1.42 * 10^{6} \mathrm{~m}^{-1}$ which is 6 orders of magnitude higher than the loss of TM mode when the waveguide has the same structure. So this waveguide can be used as a filter of the TE modes and pass the TM mode. The effective refractive indices are in the range of $0-1.3$, which is much lower than that of the silicon. This is because that the modes interaction with the metal is huge for the electric field amplitude of TE mode $\left(E_{y}\right)$ is very large.

In order to get a better understanding of the TE modes, we get the mode fields of a TE mode in the waveguide at $f=0.3 \mathrm{THz}, t=10 \mathrm{~nm}$ and $d=0.5 \mathrm{~mm}$ according to Eq. (2), as shown in Fig. 10.

At this condition, the loss of the considered mode is $445,374 \mathrm{~m}^{-1}$, which is more than 5 orders of magnitude higher than the loss of TM mode. A considerable field is 
Fig. 9 The losses (a) and the corresponding effective refractive indices (b) of TE modes in the waveguide changing as a function of $\mathrm{THz}$ frequency $f$ when $t=10 \mathrm{~nm}$ and $d=0.5 \mathrm{~mm}$

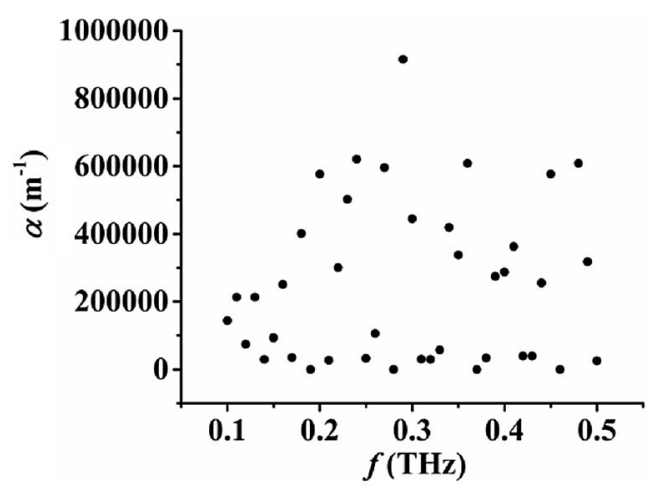

(a)

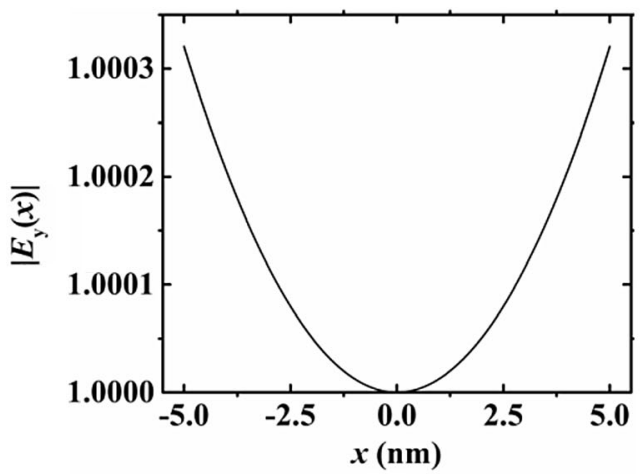

(a)

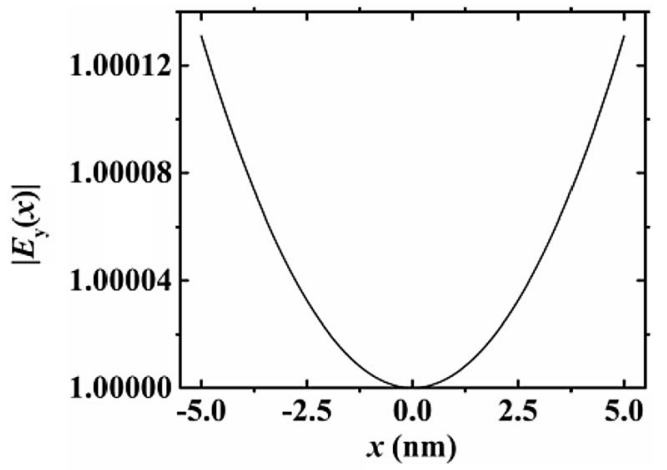

(a)

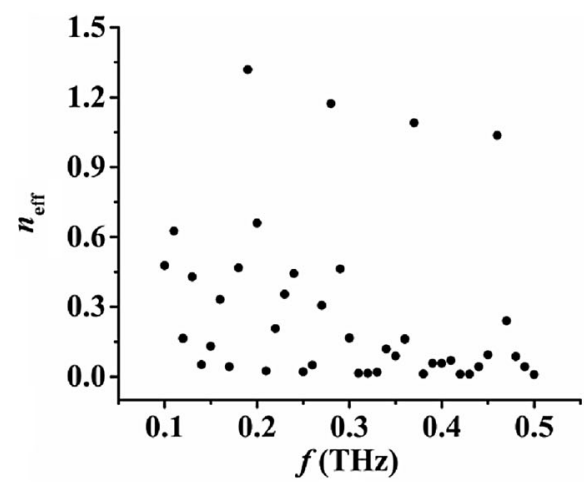

(b)

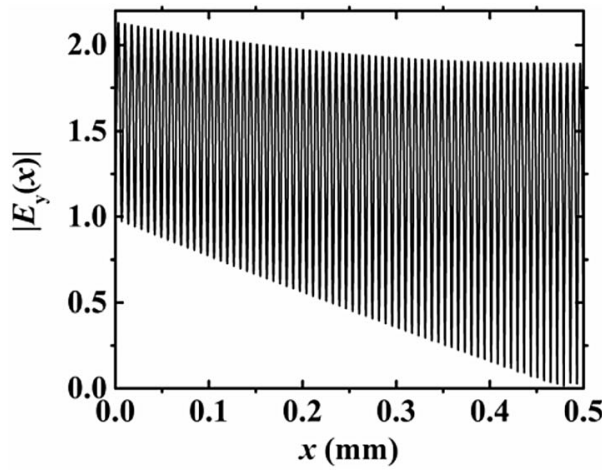

(b)

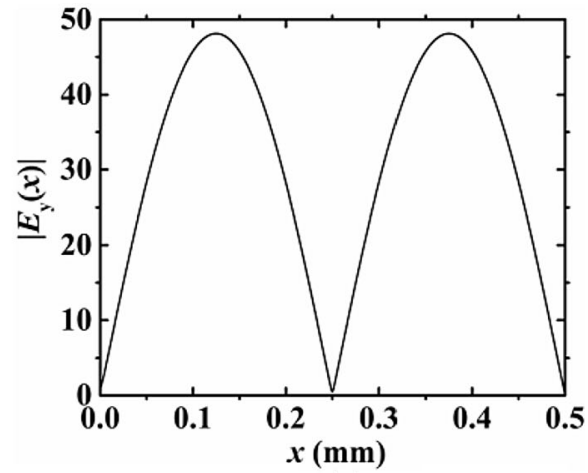

(b) distributed in the nanofilm, which causes the huge electricmetal interaction. Figure 10b shows the phase in the coated silicon slabs which is more than $50 \pi$, the order of the mode is very large.

We also calculate the field of a lower mode at $0.19 \mathrm{THz}$, as shown in Fig. 11.

At this condition the loss of the considered mode is $102.89 \mathrm{~m}^{-1}$, which is about only 2 orders of magnitude higher than the loss of TM mode. From Fig. 11, we can see the relative percentage of energy in the metal nanofilm is much lower (the field amplitude is 1 in the metal film but it is 50 in the silicon slab). The phase in the coated silicon slab is only $2 \pi$, which tells us that the order of the mode is 2.

The TE modes in the parallel-plate waveguide have low cut-off frequencies, and the losses near the low cut-off frequencies are huge. The adding of the metal film in the middle cause the low cut-off frequencies to be complex, so the losses of TE modes in the metal nanofilm-dielectricplate waveguide are huge and multiple modes appear.

When $t=10 \mathrm{~nm}$ and $f=0.5 \mathrm{THz}$, we calculate Eq. (3) and get the loss of TM mode in the waveguide as a function of silicon slab thickness $d$, as shown in Fig. 12a. As $d$ increases, the loss of TM mode decreases monotonously. The 
Fig. 12 a The loss of TM mode in the metal nanofilm-dielectricplate waveguide changing as a function of coated silicon thickness $d$ when $t=10 \mathrm{~nm}$ and $f=0.5 \mathrm{THz}$; b the loss of TM mode in the metal nanofilmdielectric-plate waveguide changing as a function of nanofilm thickness $t$ when $d=0.5 \mathrm{~mm}$ and $f=0.5 \mathrm{THz}$

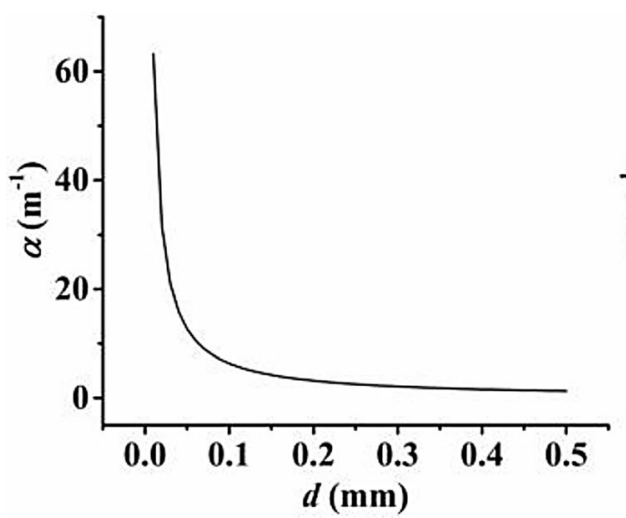

(a)

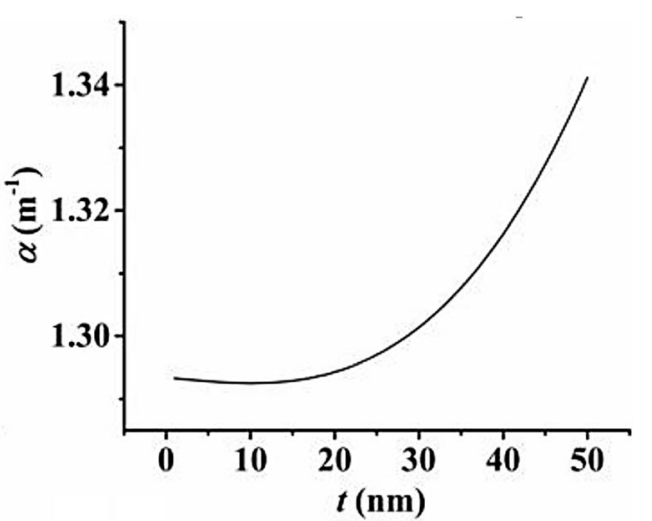

(b) loss change from $63.2 \mathrm{~m}^{-1}$ (at $d=0.01 \mathrm{~mm}$ ) to $1.29 \mathrm{~m}^{-1}$ (at $d=0.5 \mathrm{~mm}$ ). The increasing of $d$ have significance influence on the mode loss, the effects of coated silicon slabs on the TM mode are larger.

We also get the loss of TM mode in the waveguide as a function of $t$ according to Eq. (3), at $d=0.5 \mathrm{~mm}$ and $f=0.5 \mathrm{THz}$, as shown in Fig. 12b. As $t$ increases from $1 \mathrm{~nm}$ to $50 \mathrm{~nm}$, the loss increases slightly (from $1.29 \mathrm{~m}^{-1}$ at $t=1 \mathrm{~nm}$ to $1.34 \mathrm{~m}^{-1}$ at $t=50 \mathrm{~nm}$ ), which tells us the effects of the nanofilm on the TM mode are much lower. However, as discussed above, the effects of the nanofilm on the TE modes are huge.
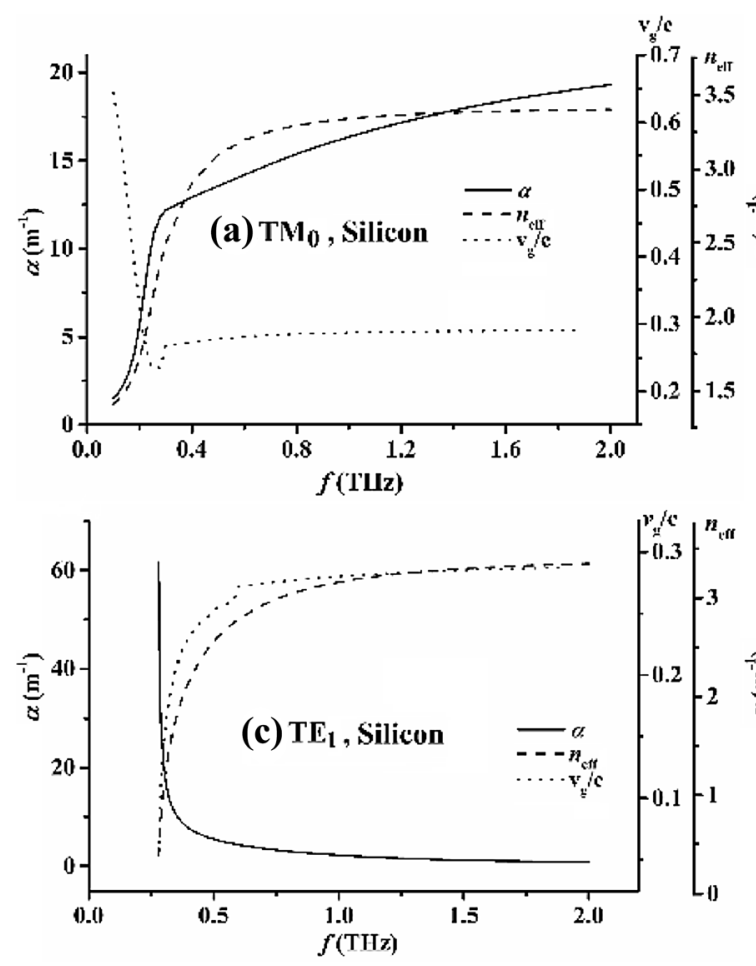

Fig. 13 The dependence of loss (solid line), effective refractive index (dashed line) and group velocity (dotted line) of the waveguide with the parameters of $t=0.1 \mathrm{~mm}, w=0.1 \mathrm{~mm}$ on THz frequency. $\mathbf{a} \mathrm{TM}_{0}$

\section{THz single-dielectric-slab-coated parallel-plate waveguide (SDSCPPW)}

\section{Mode characteristics of the waveguide}

Figure 1d shows the structure of this case. Copper is adopted as the material of the metal plates. The material of the dielectric slab is chosen as the silicon or plastic. When $t=0.1 \mathrm{~mm}, w=0.1 \mathrm{~mm}$, we calculate Eqs. (5) and (6), and get the loss (solid line) and the effective refractive index (dashed line) of the different slab materials (silicon and plastic) waveguide at different $\mathrm{THz}$ frequencies, as
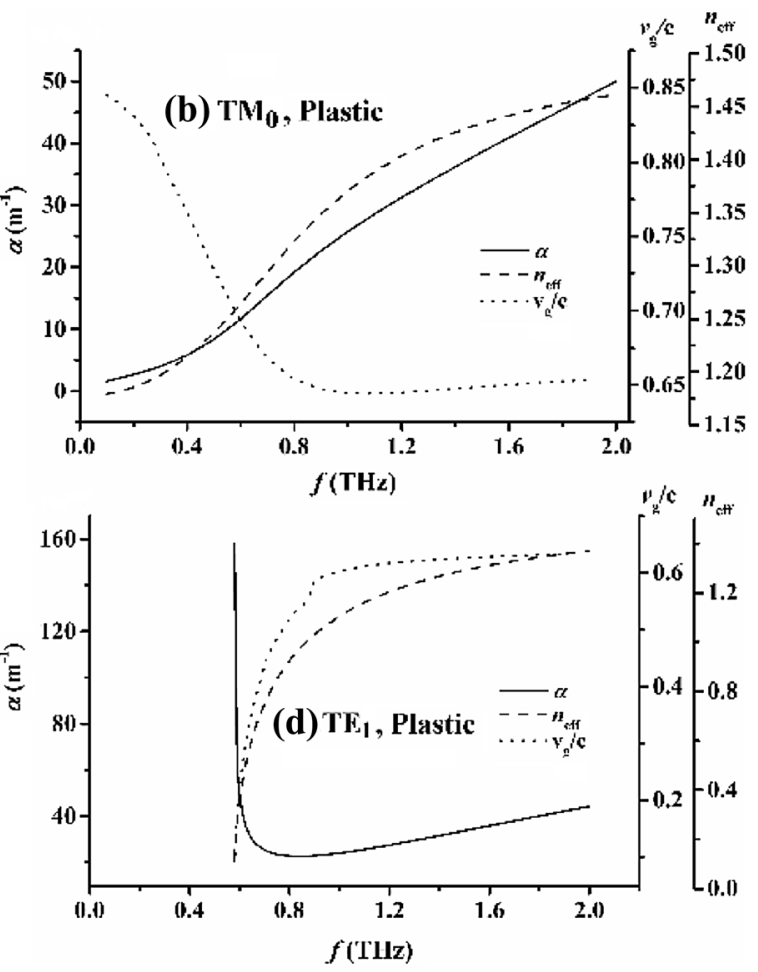

mode with a silicon slab, $\mathbf{b} \mathrm{TM}_{0}$ mode with a plastic slab, $\mathbf{c} \mathrm{TE}_{1}$ mode with a silicon slab, $\mathbf{d}$ is $\mathrm{TE}_{1}$ mode with a plastic slab 
shown in Fig. 13. The group velocity of the guiding modes is shown in dotted line.

The loss and effective refractive index of $\mathrm{TM}_{0}$ mode, which is also the transverse-electromagnetic (TEM) mode of the waveguide, are increasing monotonously. When $f$ is larger, more $\mathrm{THz}$ energy will distribute in the loss dielectric slab and less in the air interval. The loss changing law of $\mathrm{TM}_{0}$ mode is the same as described in Ref. [21]. After coating a dielectric slab, the group velocity of $\mathrm{TM}_{0}$ mode in the waveguide is only $0.23-0.64$ (for silicon slab) or 0.64-0.84 (for plastic slab) of the light velocity in vacuum. The changing slope of group velocity is much sharper in the lower frequency. For silicon slab-coated waveguide, the GVD is much larger than that of plastic slab-coated waveguide in the narrow lower frequency region, which can be seen from the less sharp and wider sharp region of the group velocity of the plastic-coated waveguide.

In the region of higher than $0.30 \mathrm{THz}$ (silicon-coated waveguide) and $0.80 \mathrm{THz}$ (plastic-coated waveguide), the GVD of the waveguide is much lower. When the GVD of the waveguide is larger, the changing of the effective refractive index is also sharper. The refractive index of silicon is 3.42 (1.5 for plastic), so the effective refractive index of silicon-coated waveguide is in the region of 1-3.42 (1-1.5 for plastic coating). After coating, the TM mode loss is in the region $1.5-50 \mathrm{~m}^{-1}$, which is only 1 order of magnitude larger than that of uncoated waveguide.

From Fig. 13c, d, we can see that there appears a low cut-off frequency for $\mathrm{TE}_{1}$ mode. Both the loss and the GVD are much larger near the low cut-off frequency. The low cut-off frequency of silicon-coated waveguide is about $0.28 \mathrm{THz}(0.58 \mathrm{THz}$ for plastic coating). According to Ref. [22], we can know the low cut-off frequency for $\mathrm{TE}_{1}$ mode of this waveguide is:

$f_{\mathrm{c}}=\frac{c}{2\left(n_{2} t+w\right)}$

where $\left(n_{2} t+w\right)$ is the optical width between the two parallel plates. We substitute the values $(t=0.1 \mathrm{~mm}$, $w=0.1 \mathrm{~mm}, c=3 * 10^{8} \mathrm{~m} / \mathrm{s}, n_{2}=3.42$ or 1.5$)$ to Eq. (12) and get $f_{\mathrm{c}}=0.34 \mathrm{THz}$ for silicon-coated waveguide, and $f_{\mathrm{c}}=0.60 \mathrm{THz}$ for plastic-coated waveguide. We can know that the low cut-off frequency is strongly affected by the refractive index of the slab.

When $f=1 \mathrm{THz}, t=0.1 \mathrm{~mm}$, we get the loss (solid line) and the effective refractive index (dashed line) of the different slab material (silicon and plastic) waveguide at the different air interval $w$, as shown in Fig. 14:

For $\mathrm{TM}_{0}$ mode, the loss increases slightly to a stable value in silicon-coated waveguide, while there is a minim loss in plastic-coated waveguide when $w$ changes from 0 to $0.1 \mathrm{~mm}$. The effective refractive index also only have slightly changes. For the same parameters of the structure, the optical width between the two parallel plates of the silicon-coated waveguide is much larger than that of the plastic-coated waveguide.

For $\mathrm{TE}_{1}$ mode at $f=1 \mathrm{THz}, t=0.1 \mathrm{~mm}$, there is a cutoff $w_{\mathrm{c}}$ for plastic-coated waveguide $\left[w_{\mathrm{c}}=0 \mathrm{~mm}\right.$ according to Eq. (12)], while it is absence for silicon-coated waveguide. We can modify the low cut-off frequency of the
Fig. 14 The dependence of loss (solid line) and effective refractive index (dashed line) of the waveguide with the parameter of $t=0.1 \mathrm{~mm}$, and frequency $f=1 \mathrm{THz}$ on air interval $w$. a $\mathrm{TM}_{0}$ mode with a silicon slab, b $\mathrm{TM}_{0}$ mode with a plastic slab, $\mathbf{c} \mathrm{TE}_{1}$ mode with a silicon slab, $\mathbf{d} \mathrm{TE}_{1}$ mode with a plastic slab
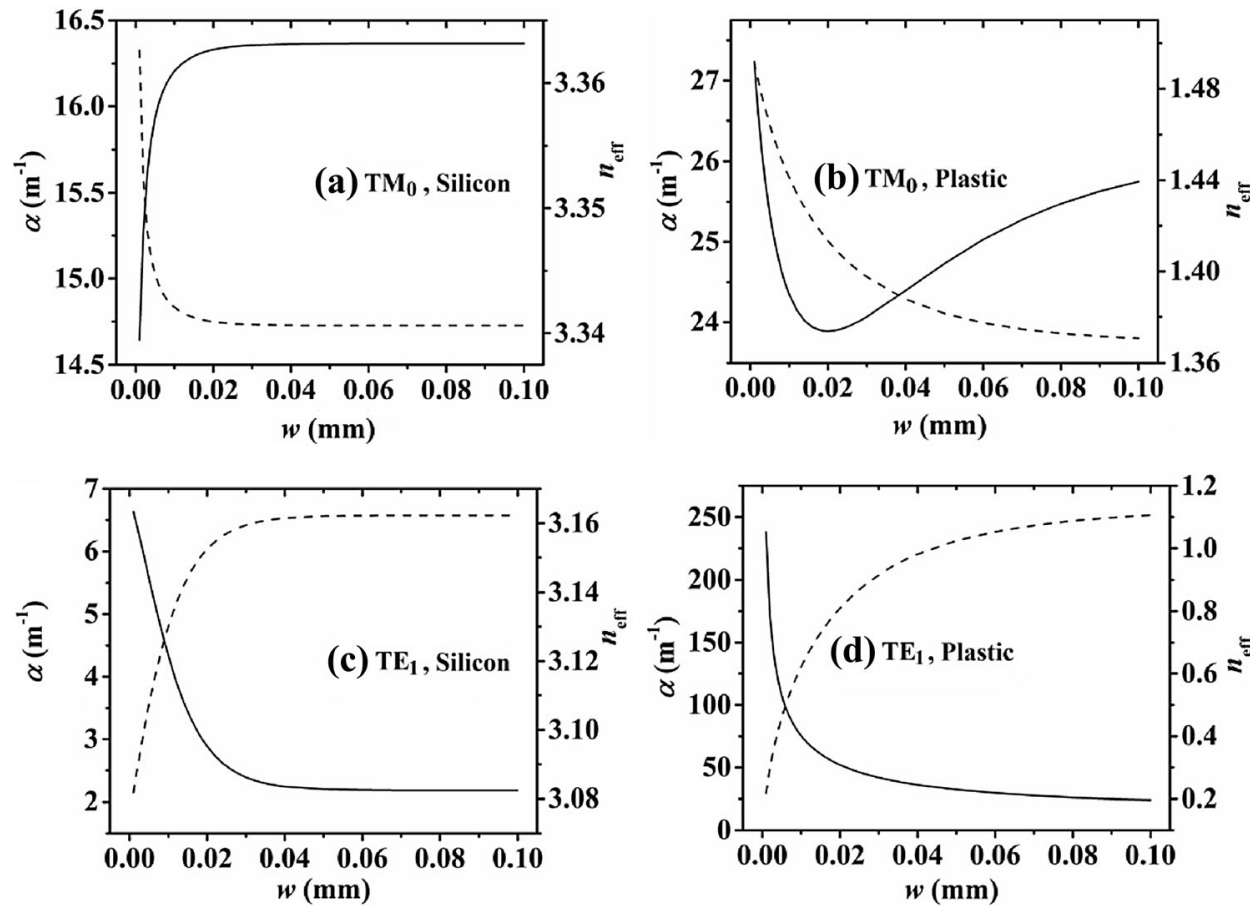
Fig. 15 The dependence of loss (solid line) and effective refractive index (dashed line) of the waveguide with the parameter of $w=0.1 \mathrm{~mm}$, and frequency $f=1 \mathrm{THz}$ on slab thickness $t$. a $\mathrm{TM}_{0}$ mode with a silicon slab, $\mathbf{b} \mathrm{TM}_{0}$ mode with a plastic slab, $\mathbf{c} \mathrm{TE}_{1}$ mode with a silicon slab, $\mathbf{d} \mathrm{TE}_{1}$ mode with a plastic slab
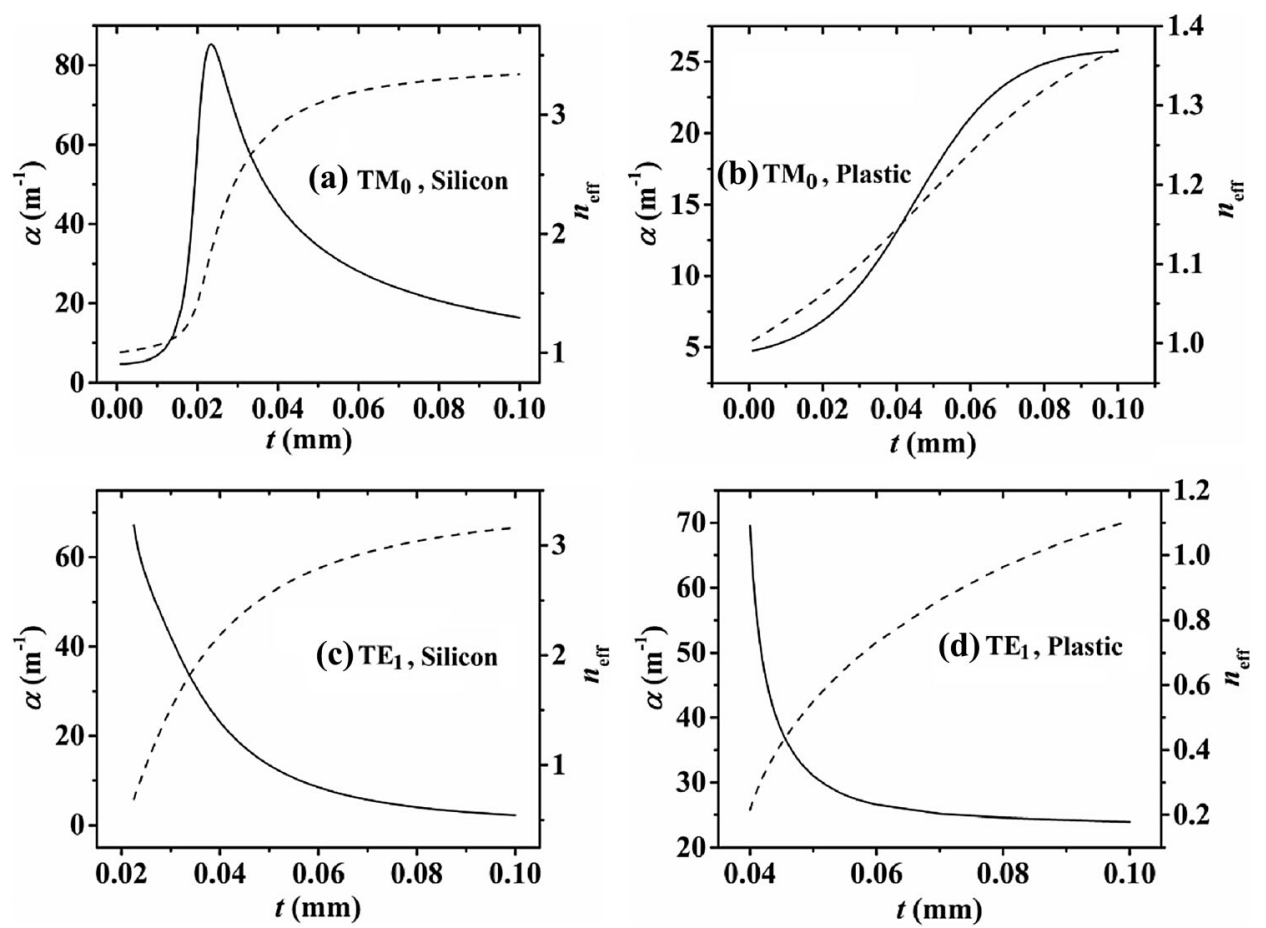

waveguide by changing the width of the air interval, which has been discussed in Ref. [20], however the sensitivity is much lower for silicon-coating waveguide.

When $f=1 \mathrm{THz}, w=0.1 \mathrm{~mm}$, we get the changing laws of loss (solid line) and the effective refractive index (dashed line) of the different slab material waveguide to $t$, as shown in Fig. 15. There is a loss peak for $\mathrm{TM}_{0}$ mode in silicon-coated waveguide while it increases monotonously for plastic-coated waveguide. The effective refractive index increases monotonously from 1 to the dielectric

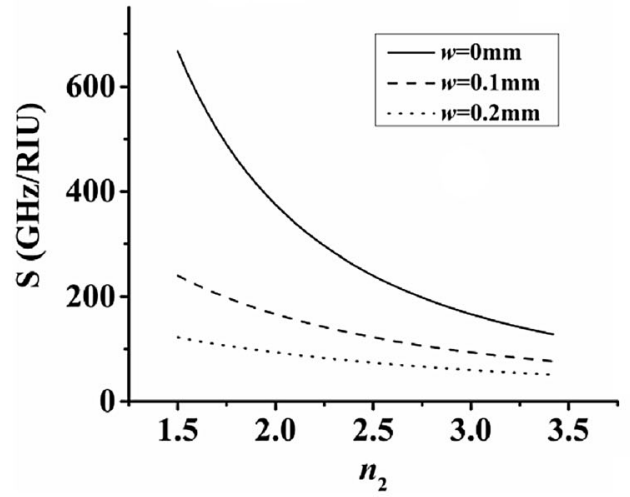

(a) refractive index, which is the evidence for more energy in the dielectric slab, and the GVD will be higher for larger $t$.

There is a low cut-off $t_{\mathrm{c}}$ for $\mathrm{TE}_{1}$ mode [it is $0.015 \mathrm{~mm}$ for silicon waveguide, and $0.033 \mathrm{~mm}$ for plastic waveguide, according to Eq. (12)]. However, when $w$ is large enough, $t_{\mathrm{c}}$ will disappear.

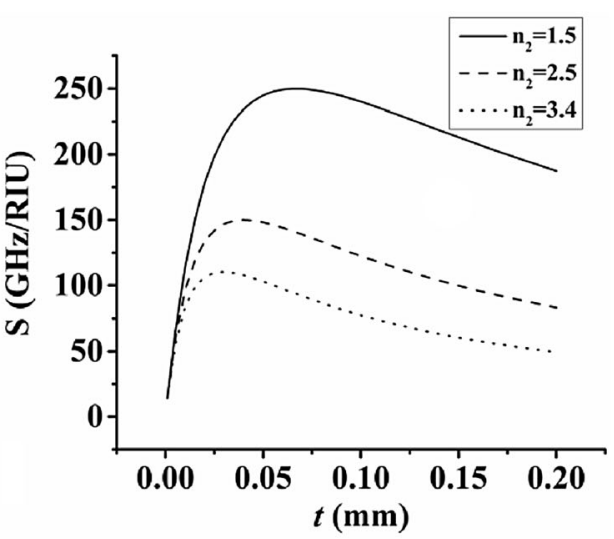

(b)

Fig. 16 a The sensitivities of $f_{\mathrm{c}}$ to refractive index $n_{2}$ of the coating slab at different values of $n_{2}$ for different waveguide structure. Solid line is at the condition of $w=0 \mathrm{~mm}$, dashed line is at the condition of $w=0.1 \mathrm{~mm}$, dotted line is at the condition of $w=0.2 \mathrm{~mm}$, and $t=0.1 \mathrm{~mm}$ for all cases, $\mathbf{b}$ the sensitivities of $f_{\mathrm{c}}$ to $n_{2}$ at different values of $t$ for different refractive index of the dielectric slab. Solid line is at the condition of $n_{2}=1.5$, dashed line is at the condition of $n_{2}=2.5$, dotted line is at the condition of $n_{2}=3.4$, and $w=0.1 \mathrm{~mm}$ for all cases 


\section{The application of refractive index sensing}

According to Eq. (12), the low cut-off frequency of $\mathrm{TE}_{1}$ mode is determined by $n_{2}, t$ and $w$. In case of fixed $t$ and $w$, the changing of $n_{2}$ will move the cut-off frequency. So the changing of $f_{\mathrm{c}}$ has a sensitivity to the changing of $n_{2}$, and it can be represented as [20]:

$S=\left|\frac{\partial f_{\mathrm{c}}}{\partial n_{2}}\right|=\frac{c t}{2\left(n_{2} t+w\right)^{2}}$

According to Eq. (13), I get the sensitivities of $f_{\mathrm{c}}$ to $n_{2}$ at different value of $n_{2}$ and $t$ for different waveguide structure, as shown in Fig. 16. We can know that the sensitivity decreases monotonously as $n_{2}$ increases, and it is larger when $w$ is smaller. When $w=0 \mathrm{~mm}$ and $n_{2}=1.5$, the highest sensitivity is up to $S=666.7 \mathrm{GHz} / \mathrm{RIU}$ (refractiveindex-unit). While there is a peak for $S$ changing to $t$. When $n_{2}$ is smaller, the peak of $S$ appears at larger $t$ (for example the peak appears at $t=0.065 \mathrm{~mm}$ for $n_{2}=1.5$, while at $t=0.03 \mathrm{~mm}$ for $n_{2}=3.4$ ).

\section{Hybrid THz SPPs in double-dielectric-slab-coated metal plate waveguide (DDSCMPW)}

Figure 1e shows the structure of this case. The materials of the coating dielectric slabs are chosen as Polystyrene (PS) and silicon. Copper is adopted as the material of metal plate. When $t=0.1 \mathrm{~mm}, a=0.5 \mathrm{~mm}$, we get the loss (a) and effective refractive index (b) of the $1-\mathrm{h}$ coated (silicon is the out-coating) metal plate waveguide and the loss [(c) solid line] and effective refractive index [(c) dashed line] of the $\mathrm{h}-1$ coated (silicon is the innercoating) metal plate waveguide as a function of the $\mathrm{THz}$ wave frequency $f$, as shown in Fig. 17.

From Fig. 17a, we can see even though the basic loss increase monotonously, there are loss nadirs at the frequencies of $0.45 \mathrm{THz}, 0.94 \mathrm{THz}, 1.42 \mathrm{THz}$ and $1.89 \mathrm{THz}$.
This is also because $\mathrm{THz}$ waves appear anti-resonant reflecting. The resonance frequency can also be predicted by Eq. (10). We substitute $c=3 * 10^{8} \mathrm{~m} / \mathrm{s}, t=0.1 \mathrm{~mm}$, $n_{3}=3.42$ and $n_{2}=1.58$ to the equation and get $f_{1}=0.49 \mathrm{THz}, \quad f_{2}=0.99 \mathrm{THz}, \quad f_{3}=1.48 \mathrm{THz} \quad$ and $f_{4}=1.98 \mathrm{THz}$, which show good agreements with the calculation results. The peaks of the corresponding effective refractive index at the resonant frequencies (as seen in Fig. 17b) tell us that much more energy come to the low loss and high index silicon. However, in the $\mathrm{h}-\mathrm{l}$ coated metal plate, the loss (as seen in Fig. 17c solid line) and effective index (as seen in Fig. 17c dashed line) increase monotonously. This is because when $n_{2}>n_{3}$, THz waves appear total internal reflecting (TIR) on the interface between the inner-coating silicon slab and the out-coating PS slab.

The THz SPPs are the modes confined on the surface of the metal plate. After double-dielectric coating, the modes become hybrid $\mathrm{THz}$ SPPs. The modes propagate in the waveguide with the effects of the two coating dielectric slabs and the metal plate. The effects of the $1-\mathrm{h}$ coating on the hybrid modes are related to the frequencies. When $f$ is closer to the resonant frequencies, the effects of the out coating is much stronger than the inner coating and the hybrid SPP modes transfer to dielectric mode. However, when $f$ is at the anti-resonant frequencies, the effects of the inner coating and the metal plate are much stronger than the out coating and the hybrid modes maintain the characteristics of the SPP-like modes. In the $\mathrm{h}-\mathrm{l}$ coating metal plate, the effects of the metal plate and the inner-coating slab are always higher than the out coating slab, and the hybrid mode is always the SPP-like mode.

We also get the corresponding group velocity of the waveguide when $t=0.1 \mathrm{~mm}, a=0.5 \mathrm{~mm}$, as a function of $f$, as shown in Fig. 18. We can see there are nadirs of the group velocity of the $1-\mathrm{h}$ coated waveguide, while the group velocity of the $h-1$ coated waveguide changes slightly. This tells us that the GVD of these two

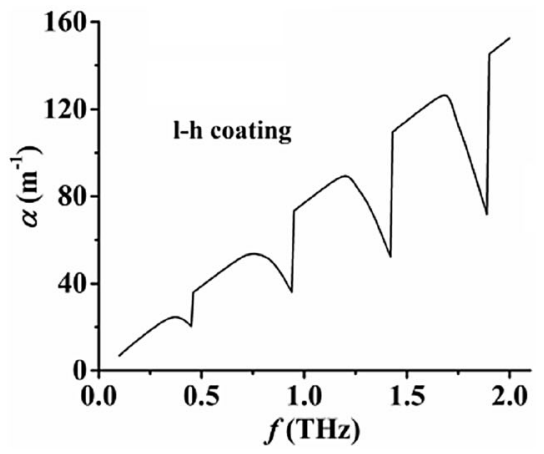

(a)

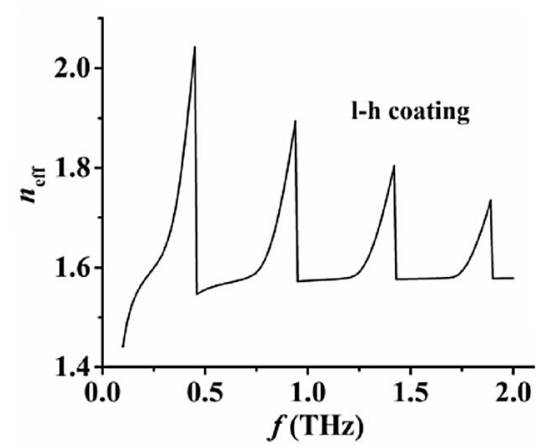

(b)

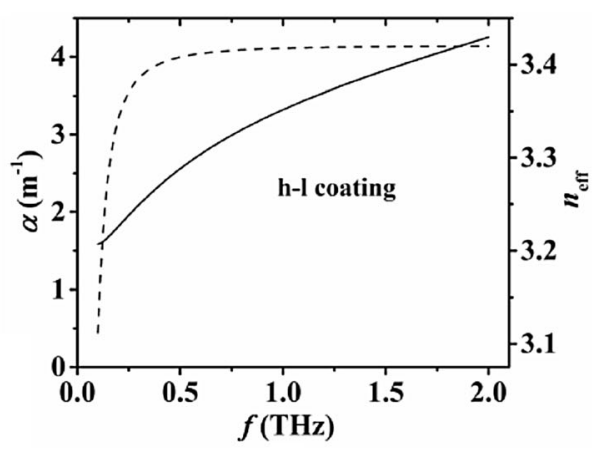

(c)

Fig. 17 The laws of the loss (a) and effective refractive index (b) of the $1-$ h coated metal plate waveguide and the loss (c solid line) and effective refractive index (c dashed line) of the h-l coated metal plate waveguide changing as a function of the THz wave frequency $f$ 
Fig. 18 The law of the group velocity changing as a function of $f$ when $t=0.1 \mathrm{~mm}$, $a=0.5 \mathrm{~mm}$ of the $1-\mathrm{h}$ coated waveguide (a) and the $\mathrm{h}-1$ coated waveguide (b)

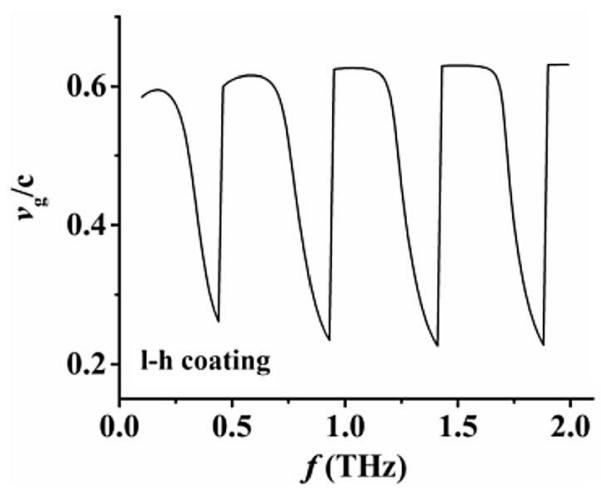

(a)

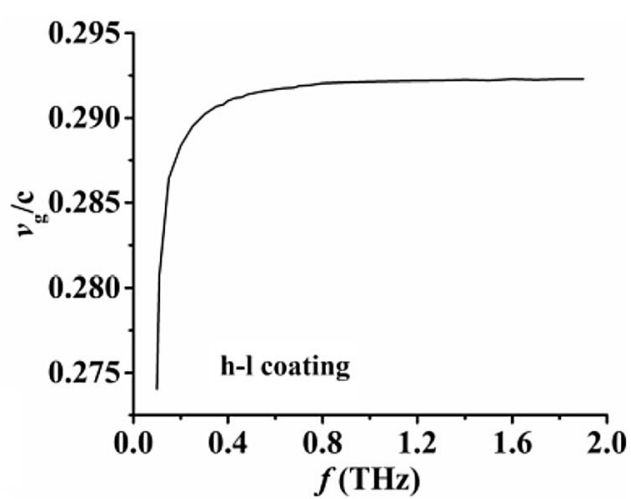

(b)

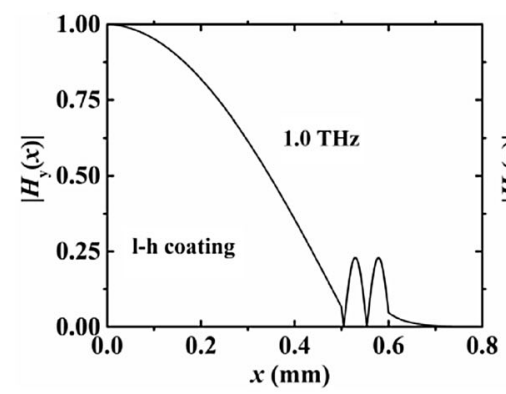

(a)

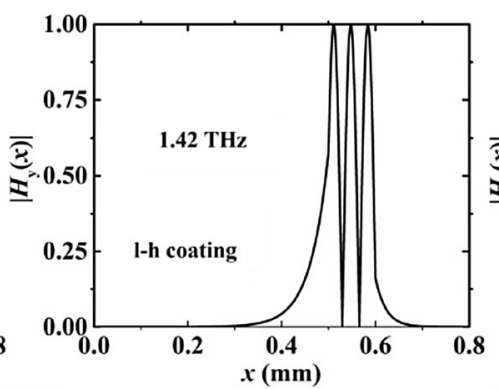

(b)

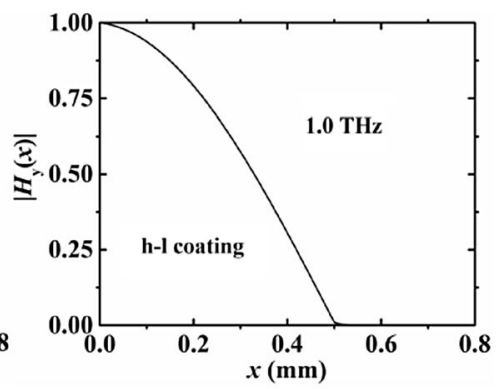

(c)

Fig. 19 The normalized mode field of $1-\mathrm{h}$ coated metal plate at the anti-resonant frequency $1.0 \mathrm{THz}(\mathbf{a})$ and resonance frequency $1.42 \mathrm{THz}(\mathbf{b})$, and the normalized mode field of $\mathrm{h}-\mathrm{l}$ coated metal plate at $1.0 \mathrm{THz}$ (c)

waveguides is totally different. The GVD of the $1-\mathrm{h}$ coated waveguide is much higher and more complex. This phenomenon is useful for the application as a THz sensor.

In order to get a better understanding of the hybrid modes, we get the mode fields of both the $1-\mathrm{h}$ coated waveguide and the $\mathrm{h}-1$ coated waveguide at $f=1.0 \mathrm{THz}$ or $1.42 \mathrm{THz}, t=0.1 \mathrm{~mm}$ and $a=0.5 \mathrm{~mm}$ according to Eq. (7), as shown in Fig. 19.

For the $1-h$ coated waveguide, we can see that at the anti-resonant frequency $1.0 \mathrm{THz}, \mathrm{THz}$ wave amplitude peak is at the metal plate interface which tells us that at this condition the metal plate still has significant effects on the hybrid modes and the hybrid mode is still SPP-like. From Fig. 19b, we can see at the resonant frequency $1.42 \mathrm{THz}$, almost no energy is distributed in the metal plate and only some in the inner-coating PS slab. Most of the energy is distributed in the out-coating silicon slab, which tells us that the hybrid SPPs modes transfer to dielectric mode totally, and the out-coating has the largest effect at this condition.

For the $\mathrm{h}-\mathrm{l}$ coated waveguide, we can see that at the frequency $1.0 \mathrm{THz}$, it appears TIR on the interface between

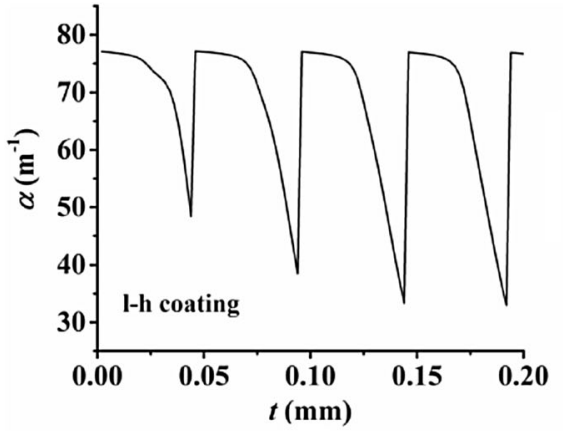

(a)

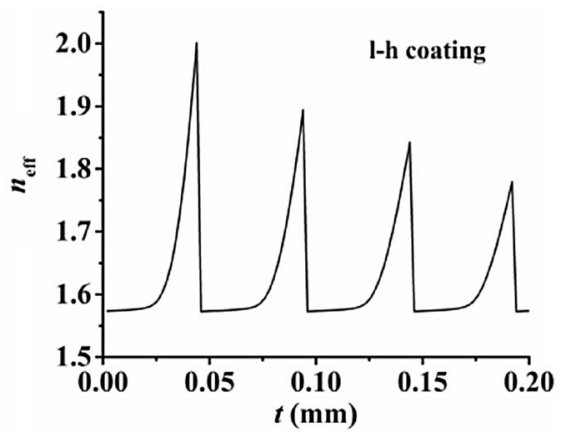

(b)

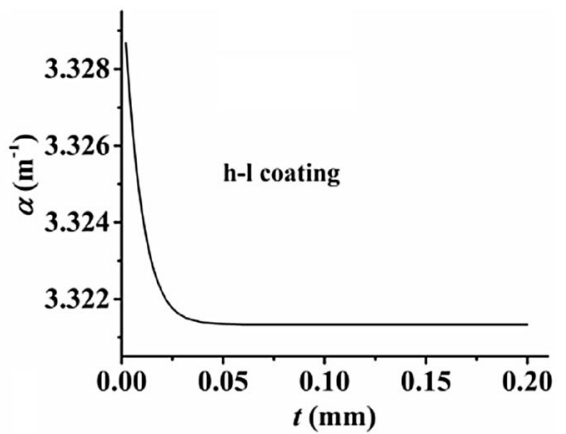

(c)

Fig. 20 The laws of the loss (a) and the corresponding effective refractive index (b) of the guiding mode in the 1-h coated metal plate and the loss in the $\mathrm{h}-1$ coated metal plate (c) changing as a function of $t$ 
Fig. 21 The laws of loss (solid line) of the $1-\mathrm{h}$ coated metal plate waveguide (a) and the $\mathrm{h}-1$ coated metal plate waveguide (b) changing as a function of $a$, the dashed line is the corresponding effective refractive index of the guiding mode

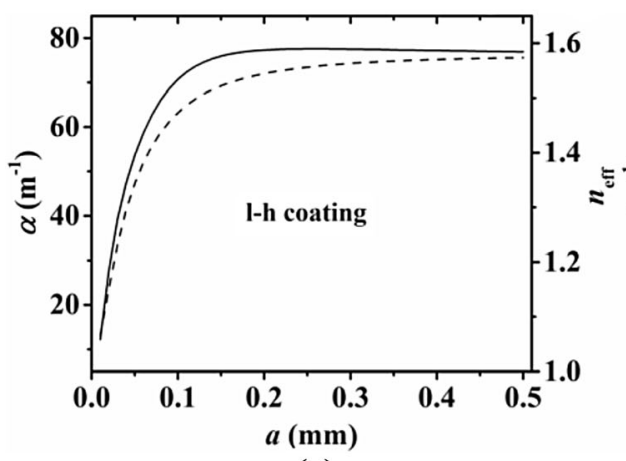

(a)

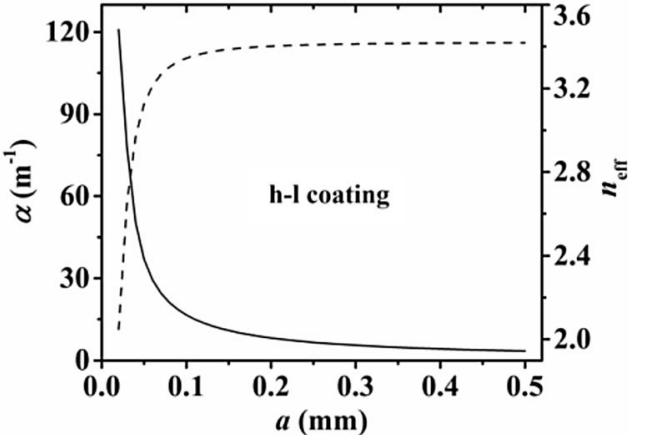

(b) the inner-coating silicon and the out-coating PS. The waves in the out-coating PS and the outside air are evanescent waves, and most of the energy is distributed in the innercoating silicon which has the largest effects on the hybrid modes. For the $\mathrm{h}-\mathrm{l}$ coating, there is a considerable energy in the metal plate at every frequency which tells us that the metal plate always has significant effects on the hybrid modes and the hybrid mode is always SPP-like.

At $f=1.0 \mathrm{THz}$ and $a=0.5 \mathrm{~mm}$, we get the loss (a) and the corresponding effective refractive index (b) of the guiding mode in the $1-\mathrm{h}$ coated metal plate and the losses in the $\mathrm{h}-1$ coated metal plate (c) as a function of $t$, as shown in Fig. 20:

We can see there are loss nadirs at the thickness of $0.044 \mathrm{~mm}, \quad 0.094 \mathrm{~mm}, \quad 0.144 \mathrm{~mm}$ and $0.192 \mathrm{~mm}$ in Fig. 20a. When $1.0 \mathrm{THz}$ becomes the resonant frequency for the out-coating silicon thickness, much more energy will be distributed in the low loss high index silicon. The resonant silicon thicknesses can be predicted by Eq. (11). We substitute $c=3 * 10^{8} \mathrm{~m} / \mathrm{s}, f=1.0 \mathrm{THz}, n_{2}=3.42$ and $n_{1}=1.58$ to the equation, and get $t_{1}=0.049 \mathrm{~mm}$, $t_{2}=0.099 \mathrm{~mm}, t_{3}=0.148 \mathrm{~mm}$ and $t_{4}=0.198 \mathrm{~mm}$. The numerical values are in good agreement with the theoretical values.

We can know the effects of the two coating dielectric slabs of the 1-h coating metal plate on the hybrid THz SPPs is strongly affected by the out coating silicon thickness. At the resonant silicon thicknesses, the effects of the out coating are much more obviously, while at the anti-resonant silicon thicknesses, the effects of the inner coating are much more obviously. However, the loss of the $\mathrm{h}-1$ coated metal plate decreases monotonously and change slightly, as can be seen in Fig. 20c, which tells us that even though the thickness of the out-coating PS slab is increasing, its effects on the hybrid modes are much smaller. This is because of the TIR on the interface.

When $f=1.0 \mathrm{THz}$ and $t=0.1 \mathrm{~mm}$, we get the loss (solid line) of the $1-\mathrm{h}$ coated metal plate waveguide (a) and the $\mathrm{h}-\mathrm{l}$ coated metal plate waveguide (b) as a function of $a$, as shown in Fig. 21. The dashed line is the corresponding effective refractive index of the guiding mode.

In Fig. 21, the loss of the $1-h$ coated waveguide increases monotonously while the loss of the $\mathrm{h}-1$ coated waveguide decreases monotonously. And the corresponding effective refractive index increases monotonously to the effective refractive index of the inner-coating material (1.58 for PS, 3.42 for silicon). At $a=t=0.1 \mathrm{~mm}$, the effective refractive index of the $1-\mathrm{h}$ coated waveguide is 1.47 , and the effective refractive index of the $\mathrm{h}-1$ coated waveguide is 3.34 , which tells us that in the range of $a>0.1 \mathrm{~mm}$, the effects of the inner coating on the hybrid THz SPPs is much more important. When $a<0.1 \mathrm{~mm}$, the effective refractive index increases sharply as $a$ increases, which tells us the effects of the inner coating increase quickly.

\section{Summary}

In summary, we have presented five kinds of layered $\mathrm{THz}$ waveguides. The modified $\mathrm{THz}$ SPPs on DSCMFW is discussed in detail. For TLDSW, we find that the waveguide with high-low-high refractive indices guide $\mathrm{THz}$ wave as the anti-resonant reflecting mechanism. The mode characteristics of both TM mode and TE mode in MNDPW are presented. The application of a TE modes filter for this waveguide is put forward. The GVD of $\mathrm{TM}_{0}$ mode in SDSCPPW is discussed in detail. For $\mathrm{TE}_{1}$ mode, the characteristics of the low cut-off frequency are given. The low cut-off frequency have a sensitivity $(S)$ to the refractive index of the dielectric slab. The highest $S$ can be 666.7 GHz/RIU when $n_{2}=1.5, w=0$ and $t=0.1 \mathrm{~mm}$. Moreover, the hybrid THz SPPs on DDSCMPW have been presented. At the resonant frequencies and resonant silicon thicknesses, the hybrid SPP modes transfer to dielectric modes. However, for the $\mathrm{h}-1$ coating, the hybrid mode is always a SPP-like mode. We believe that these results are very useful for designing of $\mathrm{THz}$ waveguides, sensors, and 
filters, and for modified and hybrid $\mathrm{THz}$ SPPs in waveguides.

Acknowledgements The authors would like to acknowledge financial supports from the Engineering and Physical Sciences Research Council (EPSRC) on Grant "Graphene Flexible Electronics and Optoelectronics" (EP/K01711X/1) and the EU Graphene Flagship (FP7-ICT-604391).

Open Access This article is distributed under the terms of the Creative Commons Attribution 4.0 International License (http://crea tivecommons.org/licenses/by/4.0/), which permits unrestricted use, distribution, and reproduction in any medium, provided you give appropriate credit to the original author(s) and the source, provide a link to the Creative Commons license, and indicate if changes were made.

\section{References}

1. K. Wang, D.M. Mittleman, Metal wires for terahertz waveguiding. Nature 432, 376 (2004)

2. J. Liu, H. Liang, M. Zhang, H. Su, Metal plate for guiding terahertz surface plasmon-polaritons and its sensing applications. Opt. Commun. 339, 222-227 (2015)

3. X.F. Zhang, L.F. Shen, J.-J. Wu, T.-J. Yang, Terahertz surface plasmon polaritons on a periodically structured metal film with high confinement and low loss. J. Electromagn. Waves Appl. 23, 2451-2460 (2009)

4. K. Wang, D.M. Mittleman, Dispersion of surface plasmon polaritons on metal wires in the terahertz frequency range. Phys. Rev. Lett. 96, 157401 (2006)

5. Q. Cao, J. Jahns, Azimuthally polarized surface plasmons as effective terahertz waveguides. Opt. Express 13, 511-518 (2005)

6. M. Gong, T.-I. Jeon, D. Grischkowsky, THz surface wave collapse on coated metal surfaces. Opt. Express 17, 17088-17101 (2009)

7. J. Saxler, J.G. Rivas, C. Janke, H.P.M. Pellemans, P.H. Bolívar, H. Kurz, Time-domain measurements of surface plasmon polaritons in the terahertz frequency range. Phys. Rev. B 69, 155427 (2004)

8. T.H. Isaac, W.L. Barnes, E. Hendry, Determining the terahertz optical properties of subwavelength films using semiconductor surface plasmons. Appl. Phys. Lett. 93, 241115 (2008)

9. Sommerfeld, Ueber die Fortpflanzung elektrodynamischer Wellen längs eines Drahtes. Ann. Phys. Chem. 67, 233-290 (1899)

10. H. Liang, S. Ruan, M. Zhang, H. Su, I.L. Li, Modified surface plasmon polaritons for the nanoconcentration and long-range propagation of optical energy. Sci. Rep. 4(5015), 1-4 (2014)

11. H. Liang, S. Ruan, S. Xu, M. Zhang, H. Su, I.L. Li, Modified surface plasmon polaritons with ultrahigh figures of merit on metal-gap-dielectric waveguides. Appl. Phys. Express 7, 122001 (2014)

12. H. Liang, S. Ruan, M. Zhang, H. Su, I.L. Li, Characteristics of modified surface plasmon polaritons on double-coated metal nanofilms. Laser Phys. Lett. 11, 115003 (2014)
13. R.F. Oulton, V.J. Sorger, D.A. Genov, D.F.P. Pile, X. Zhang, A hybrid plasmonic waveguide for subwavelength confinement and long-range propagation. Nat. Photonics 2, 496-500 (2008)

14. M.Z. Alam, J. Meier, J.S. Aitchison, M. Mojahedi, Propagation characteristics of hybrid modes supported by metal-low-high index waveguides and bends. Opt. Express 18, 12971-12979 (2010)

15. G. Gallot, S.P. Jamison, R.W. McGowan, D. Grischkowsky, Terahertz waveguides. J. Opt. Soc. Am. B 17, 851 (2000)

16. S. Atakaramians, S. Afshar, T.M. Monro, D. Abbott, Terahertz dielectric waveguides. Adv. Opt. Photon. 5, 169-215 (2013)

17. M. Nagel, A. Marchewka, H. Kurz, Low-index discontinuity terahertz waveguides. Opt. Express 14, 9944 (2006)

18. J. Liu, H. Liang, M. Zhang, H. Su, Broadband terahertz transmission within the symmetrical plastic film coated parallel-plate waveguide. Appl. Opt. 53, 6008 (2014)

19. J. Liu, H. Liang, M. Zhang, H. Su, Coupling of Sommerfeld waves using odd TM mode of double-dielectric-slab waveguide. J. Opt. 44, 53 (2014)

20. J. Liu, H. Liang, M. Zhang, H. Su, THz wave transmission within the metal film coated double-dielectric-slab waveguides and the tunable filter application. Opt. Commun. 351, 103 (2015)

21. R. Mendis, D.M. Mittleman, Comparison of the lowest-order transverseelectric $\left(\mathrm{TE}_{1}\right)$ and transverse-magnetic (TEM) modes of the parallel-plate waveguide for terahertz pulse applications. Opt. Express 17, 14839 (2009)

22. R. Mendis, D.M. Mittleman, An investigation of the lowest-order transverse-electric $\left(\mathrm{TE}_{1}\right)$ mode of the parallel-plate waveguide for THz pulse propagation. J. Opt. Soc. Am. B 26, A6 (2009)

23. R. Mendis, A. Nag, F. Chen, D.M. Mittleman, A tunable universal terahertz filter using artificial dielectrics based on parallelplate waveguides. Appl. Phys. Lett. 97, 131106 (2010)

24. J. Liu, H. Liang, M. Zhang, H. Su, Terahertz wave transmission within metal-clad anti-resonant reflecting hollow waveguides. Appl. Opt. 54, 4549 (2015)

25. J.-Y. Lu, H.-Z. Chen, C.-H. Lai, H.-C. Chang, B. You, T.-A. Liu, J.-L. Peng, Application of metal-clad anti-resonant reflecting hollow waveguides to tunable terahertz notch filter. Opt. Express 19, 162 (2011)

26. B.S. Phillips, P. Measor, Y. Zhao, H. Schmidt, A.R. Hawkins, Optofluidic notch filter integration by lift-off of thin films. Opt. Express 18, 4790 (2010)

27. R. Mendis, D. Grischkowsky, THz interconnect with low-loss and low-group velocity dispersion. IEEE Microw. Wirel. Compon. Lett. 11(11), 444-446 (2001)

28. A. Yariv, Optical Electronics in Modern Communications (Oxford University Press, Oxford, 2007)

29. M.A. Ordal, R.J. Bell, R.W. Alexander Jr., L.L. Long, M.R. Querry, Optical properties of fourteen metals in the infrared and far infrared: $\mathrm{Al} \mathrm{Co}, \mathrm{Cu}, \mathrm{Au}, \mathrm{Fe}, \mathrm{Pb}, \mathrm{Mo}, \mathrm{Ni}, \mathrm{Pd}, \mathrm{Pt}, \mathrm{Ag}, \mathrm{Ti}, \mathrm{V}$, and W. Appl. Opt. 24, 4493-4499 (1985)

30. J.R. Birch, The far-infrared optical constants of polypropylene, PTFE, and polystyrene. Infrared Phys. 33(1), 33-38 (1992)

Publisher's Note Springer Nature remains neutral with regard to jurisdictional claims in published maps and institutional affiliations. 